\title{
Further results on edge even graceful labeling of the join of two graphs
}

\author{
Mohamed R. Zeen El Deen ${ }^{1 *}$ (i) and Nora A. Omar² (D)
}

\author{
*Correspondence: \\ mohamed.zeeneldeen@suezuniv.edu.e \\ 'Department of Mathematics, \\ Faculty of Science, Suez University, \\ Suez, Egypt \\ Full list of author information is \\ available at the end of the article
}

\begin{abstract}
In this paper, we investigated the edge even graceful labeling property of the join of two graphs. A function $f$ is called an edge even graceful labeling of a graph $G=(V(G), E(G))$ with $p=|V(G)|$ vertices and $q=|E(G)|$ edges if $f: E(G) \rightarrow$ $\{2,4, \ldots, 2 q\}$ is bijective and the induced function $f^{*}: V(G) \rightarrow\{0,2,4, \cdots, 2 q-2\}$, defined as $f^{*}(x)=\left(\sum_{x y \in E(G)} f(x y)\right) \bmod (2 k)$, where $k=\max (p, q)$, is an injective function. Sufficient conditions for the complete bipartite graph $K_{m, n}=m K_{1}+n K_{1}$ to have an edge even graceful labeling are established. Also, we introduced an edge even graceful labeling of the join of the graph $K_{1}$ with the star graph $K_{1, n}$, the wheel graph $W_{n}$ and the sunflower graph $s f_{n}$ for all $n \in \mathbb{N}$. Finally, we proved that the join of the graph $\bar{K}_{2}$ with the star graph $K_{1, n}$, the wheel graph $W_{n}$ and the cyclic graph $C_{n}$ are edge even graceful graphs.
\end{abstract}

Keywords: Complete bipartite graph, Wheel graph, Sunflower graph, Edge even graceful labeling, Join of two graphs

Mathematics Subject Classification: 05 C 78, 05 C 76, 05 C 90, 05 C 99

\section{Introduction}

A labeling of a graph is a mapping that carries graph elements (edges or vertices, or both) to positive integers subject to certain constraints. Recently, graph labeling has much attention from different researches in graph theory because it has rigorous applications in many disciplines, e.g., coding theory, X-ray, radar, communication networks, circuit design, astronomy, communication network addressing, and graph decomposition problems. For more interesting applications of graph labeling, see [1-3].

In graph theory, one can generate many new graphs from given ones by using graph operation. For a graph $G$, let $q=|E(G)|$ be the cardinality of $E(G)$ and $p=|V(G)|$ be that of $V(G)$. Let $G$ and $H$ be two graphs with no vertex in common. The join of $G$ and $H$, denoted by $G+H$, defined to be the graph with vertex set and edge set given as follows: $V(G+H)=V(G) \cup V(H), E(G+H)=E(G) \cup E(H) \cup\left\{x_{1} x_{2}: x_{1} \in V(G), x_{2} \in V(H)\right\}$. If $G$ and $H$ are $\left(p_{1}, q_{1}\right)$ and $\left(p_{2}, q_{2}\right)$ graphs, respectively, then the number of vertices and edges in the join graph are $p_{1}+p_{2}$ and $q_{1}+q_{2}+p_{1} p_{2}[1]$.

Elsonbaty and Daoud [4] introduced a new type of labeling of a graph $G$ with $p$ vertices and $q$ edges called an edge even graceful labeling if there is a bijection $f$ from the edges of the graph to the set $\{2,4, \cdots, 2 q\}$ such that, when each vertex is assigned the sum of all edges incident to it $\bmod 2 k$ where $k=\max (p, q)$, the resulting vertex labels are distinct.

(c) The Author(s). 2020 Open Access This article is licensed under a Creative Commons Attribution 4.0 International License, which permits use, sharing, adaptation, distribution and reproduction in any medium or format, as long as you give appropriate credit to the original author(s) and the source, provide a link to the Creative Commons licence, and indicate if changes were made. The images or other third party material in this article are included in the article's Creative Commons licence, unless indicated otherwise in a credit line to the material. If material is not included in the article's Creative Commons licence and your intended use is not permitted by statutory regulation or exceeds the permitted use, you will need to obtain permission directly from the copyright holder. To view a copy of this licence, visit http://creativecommons.org/licenses/by/4.0/. 
The graph that admits edge even graceful labeling is called an edge even graceful graph. They introduced some path and cycle-related graphs which are edge even graceful, then Zeen El Deen [5] studied more graphs having an edge even graceful labeling.

Furthermore, Elsonbaty and Daoud [6] investigate edge even graceful labeling of cylinder grid graphs also, Daoud [7] studied the edge even graceful labeling of Polar grid graphs after that, Zeen El Deen and Omar N. [8] extended the edge even graceful labeling into $r$ - edge even graceful labeling. For a summary of graph labeling, we refer to the dynamic survey by Gallian [9].

It should be noted that the join graph is not necessarily an edge even graceful graph. For example, the wheel graph $W_{3}=K_{1}+C_{3}$ is not an edge even graceful graph. In [4], they proved that the fan graph $F_{n}=K_{1}+P_{n} ; n \geq 2$ and $W_{n}=K_{1}+C_{n} ; n>3$ are edge even graceful graphs. Now, we will study the edge even graceful labeling of the join of the graph $K_{1}$ with the star graph $K_{1, n}$, the wheel graph $W_{n}$, and the sunflower graph $s f_{n}$. Also, we will study the edge even graceful labeling of the complete bipartite graph $K_{m, n}=m K_{1}+n K_{1}$. Since the double fan graph $F_{2, n}=\bar{K}_{2}+P_{n} ; n \geq 2$ is an edge even[5], so we will study the edge even graceful labeling of the join of the graph $\bar{K}_{2}$ with the graphs $K_{1, n}, C_{n}$ and $W_{n}$.

\section{Edge even graceful labeling of the graph $K_{n, n}=n k_{\mathbf{1}}+n K_{\mathbf{1}}$}

Theorem 1 The complete bipartite graph $K_{n, n}=n K_{1}+n K_{1}$ has an edge even graceful labeling when $n>1$ is an odd number.

Proof Let us use the standard notation $p=\left|V\left(K_{n, n}\right)\right|=2 n$ and $q=\left|E\left(K_{n, n}\right)\right|=n^{2}$. The vertices of $K_{n, n}$ were divided into two disjoint sets $\left\{v_{1}, v_{2}, \cdots, v_{n}\right\}\left\{u_{1}, u_{2}, \cdots, u_{n}\right\}$ such that every pair of graph vertices in the two sets are adjacent. There are three cases:

Case (1) If $n \equiv 3(\bmod 4), n>3$, we define the function $f: E\left(K_{n, n}\right) \longrightarrow\left\{2,4, \cdots, 2 n^{2}\right\}$ as follows:

If $i$ is an odd number, $1 \leq i \leq n$

$$
f\left(u_{i} v_{j}\right)= \begin{cases}(i-1) n+j+1 & \text { if } j=1,3, \cdots, n ; \\ 2 n^{2}-[(i-1) n+j] & \text { if } j=2,4, \cdots, n-1,\end{cases}
$$

and if $i$ is an even number, $2 \leq i \leq n-1$

$$
f\left(u_{i} v_{j}\right)= \begin{cases}(i-1) n+j+2 & \text { if } j=1,3, \cdots, n-2 \\ 2 n^{2}-[(i-1) n+j+1] & \text { if } j=2,4, \cdots, n-1 \\ 2 n^{2}-[(i-1) n+1] & \text { if } j=n\end{cases}
$$

The following matrix $X=\left(a_{i j}\right)$ shows the methods of labeling, where $a_{i j}$ represents the label of the edge $u_{i} v_{j}$.

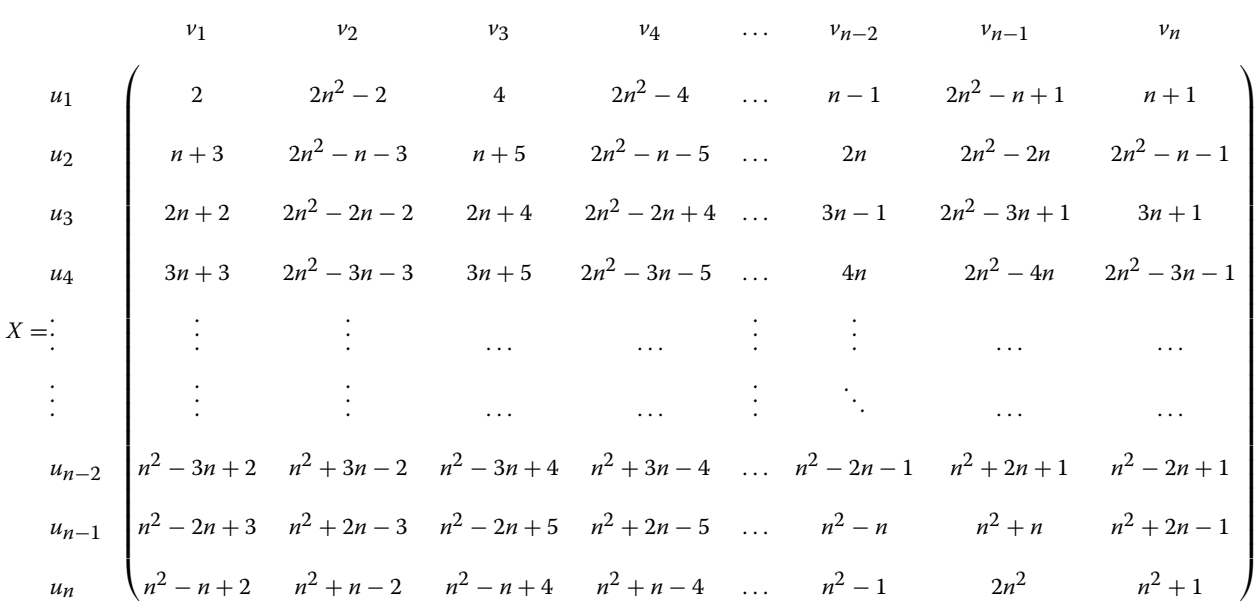


In this case, the equality of the labeling of the two vertices $u_{n}$ and $v_{n}$ forces us to change the labels of the two edges $u_{n} v_{n-1}$ and $u_{n} v_{n}$, that is, $f\left(u_{n} v_{n-1}\right)=2 n^{2}$ and $f\left(u_{n} v_{n}\right)=n^{2}+1$. Thus, the induced vertex labels are

(i) The labels of the vertices $u_{i}, i=1,2, \cdots, n$ is the sum of rows in the matrix, i.e.,

$f^{*}\left(u_{i}\right)=\left[\sum_{j=1}^{n-1} f\left(u_{i} v_{j}\right)\right] \bmod \left(2 n^{2}\right)=f\left(u_{i} v_{n}\right)$, so the labels of the vertices $u_{1}, u_{2}, u_{3}, u_{4}, \cdots, u_{n-2}, u_{n-1}$ are

$n+1,2 n^{2}-n-1,3 n+1,2 n^{2}-3 n-1, \cdots, n^{2}-2 n+1, n^{2}+2 n-1$, respectively, and $f^{*}\left(u_{n}\right)=0$.

(ii) The labels of the vertices $v_{i}$ is the sum of columns in the matrix and since $n \equiv 3(\bmod 4) \Rightarrow n=3+4 k \Rightarrow 2 q=2 n^{2}=32 k^{2}+48 k+18$, then, we have

$$
f^{*}\left(v_{i}\right)= \begin{cases}\frac{2 n^{2}+(2 i+3) n-1}{2} & \text { if } i=1,3, \cdots, n-2 ; \\ \frac{2 n^{2}-(2 i+1) n+1}{2} & \text { if } i=2,4, \cdots, n-3 .\end{cases}
$$

Hence, the labels of the vertices $v_{1}, v_{2}, v_{3}, v_{4}, \cdots, v_{n-3}, v_{n-2}$ are $\frac{2 n^{2}+5 n-1}{2}, \frac{2 n^{2}-5 n+1}{2}, \frac{2 n^{2}+9 n-1}{2}, \frac{2 n^{2}-9 n+1}{2}, \cdots, \frac{5 n+1}{2}, \frac{4 n^{2}-n-1}{2}$, respectively.

Also $f^{*}\left(v_{n}\right)=\left[\sum_{i=1}^{n} f\left(u_{i} v_{n}\right)\right] \bmod \left(2 n^{2}\right)=f\left(u_{n} v_{n}\right)=n^{2}+1$ and

$$
f^{*}\left(v_{n-1}\right)=\left[\frac{-n^{3}+n^{2}+n-1}{2}\right] \bmod \left(2 n^{2}\right)=\left[\frac{-2 n^{2}+n-1}{2}\right] \bmod \left(2 n^{2}\right)=\frac{n^{2}+n-1}{2} .
$$

Case (2) If $n=3$, the graph $K_{3,3}$ is an edge even graceful graph, see the following Fig. 1.

Case (3) If $n \equiv 1(\bmod 4)$. The labels of the edges incident to the vertices $\left\{u_{i}, i=1,2, \cdots, n-1\right\}$ are similar to the first case, but there are some changes in the label of the edges in the last row in the matrix. The labels of the edges $u_{n} v_{1}, u_{n} v_{2}, u_{n} v_{3}, u_{n} v_{4}, \cdots, u_{n} v_{\frac{n-3}{2}}, u_{n} v_{\frac{n-1}{2}}$ are given by $(n-1) n+$ $2,2 n^{2}-[(n-1) n+2],(n-1) n+4,2 n^{2}-[(n-1) n+4], \cdots, n(n-1)+$ $\left(\frac{n-1}{2}\right), 2 n^{2}-\left[n(n-1)+\left(\frac{n-1}{2}\right)\right]$ and the edge $u_{n} v_{\frac{n+1}{2}}$ label by $2 n^{2}$. Finally, we swap the direction of labeling to start labels from the end of the row, so the edges $u_{n} v_{n}, u_{n} v_{n-1}, u_{n} v_{n-2}, u_{n} v_{n-3}, \cdots, u_{n} v_{\frac{n+5}{2}}, u_{n} v_{\frac{n+3}{2}}$ will label by $(n-1) n+$

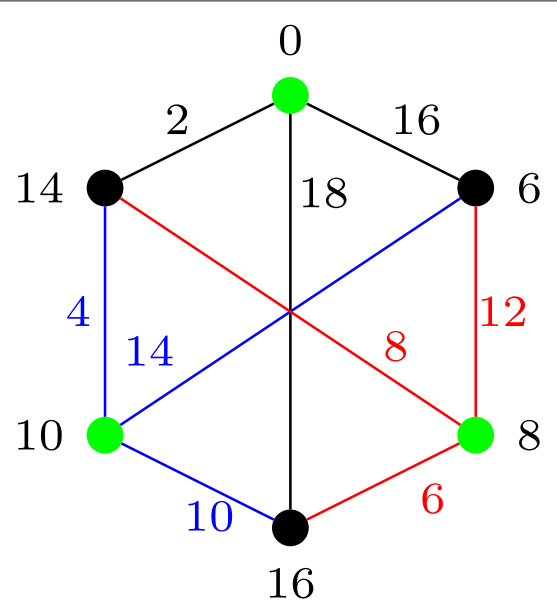

Fig. 1 An edge even graceful labeling of the graph $K_{3,3}$ 
$\frac{n+3}{2}, 2 n^{2}-\left[(n-1) n+\frac{n+3}{2}\right],(n-1) n+\frac{n+7}{2}, 2 n^{2}-\left[(n-1) n+\frac{n+7}{2}\right], \cdots,(n+$

1) $(n-1), 2 n^{2}-[(n+1)(n-1)]$ as shown in the following matrix $X=\left(a_{i j}\right)$.

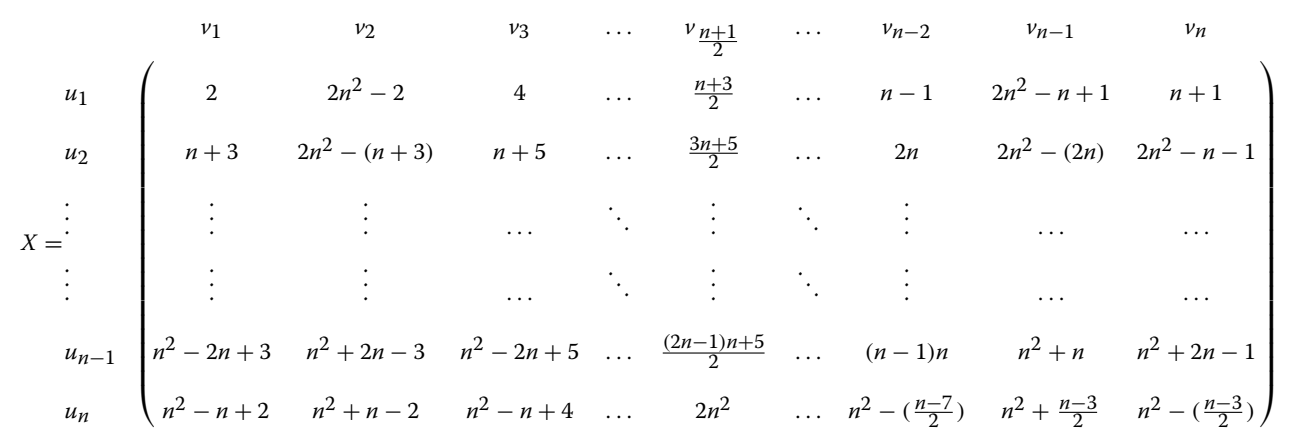

The algorithm for the matrix $X=\left(a_{i j}\right)$ of the graph $K_{n, n}$ when $n \equiv 1(\bmod 4)$ is shown below.

$n$ is odd number.

$X \rightarrow$ square matrix $[n \times n]$.

for $i 1 \longrightarrow n$,

for $j 1 \longrightarrow n$,

if $i \longrightarrow$ odd \& $i \leq n-2$.

if $j \longrightarrow$ odd,

$X(i, j)=(i-1) n+j+1$.

else if $j \longrightarrow$ even ,

$X(i, j)=2 n^{2}-[(i-1) n+j]$.

if $i==n$,

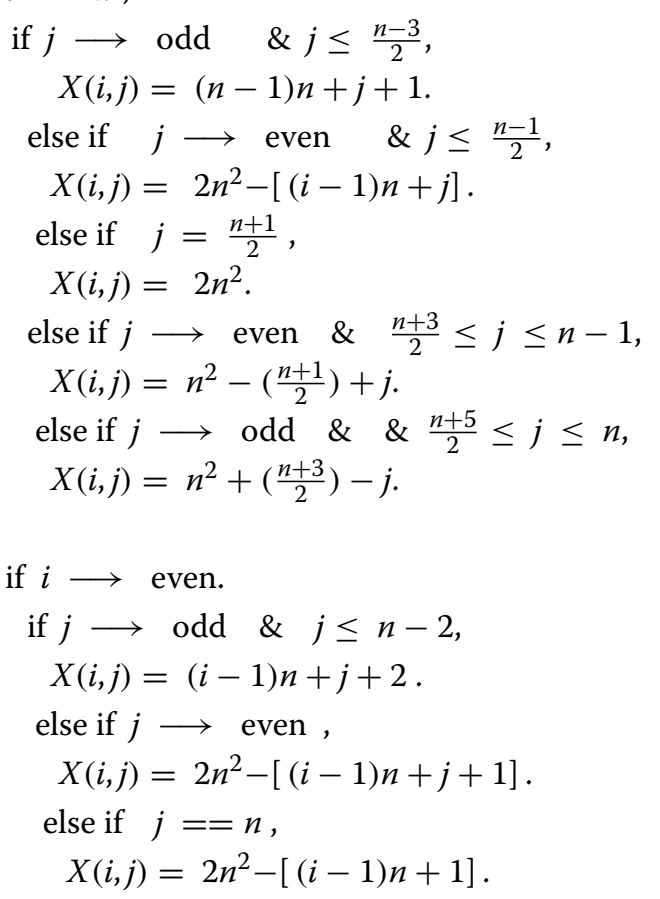

Thus, the induced vertex labels are 
(i) The labels of the vertices $u_{i}, i=1,2, \cdots, n$ is the sum of rows in the matrix, so the labels of these vertices are

$n+1,2 n^{2}-n-1,3 n+1,2 n^{2}-3 n-1, \cdots, n^{2}-2 n+1, n^{2}+2 n-1,0$, respectively.

(ii) The labels of the vertices $v_{i}$ is the sum of columns in the matrix and since $n \equiv 1(\bmod 4) \Rightarrow n=1+4 k \Rightarrow 2 q=2 n^{2}=32 k^{2}+16 k+2$, then, we have

$$
f^{*}\left(v_{i}\right)= \begin{cases}\frac{(2 i+3) n-1}{2} & \text { if } i=1,3, \cdots, \frac{n-3}{2} \\ \frac{4 n^{2}-(2 i+1) n+1}{2} & \text { if } i=2,4, \cdots, \frac{n-1}{2} .\end{cases}
$$

Hence, the labels of the vertices $v_{1}, v_{2}, v_{3}, v_{4}, \cdots, v_{\frac{n-3}{2}}, v_{\frac{n-1}{2}}$ are $\frac{5 n-1}{2}, \frac{4 n^{2}-5 m+1}{2}, \frac{9 n-1}{2}, \frac{4 n^{2}-9 m+1}{2}, \cdots, \frac{n^{2}+1}{2}, \frac{3 n^{2}-1}{2}$, respectively.

Also, $f^{*}\left(v_{\frac{n+1}{2}}\right)=\left[\frac{n^{3}-2 n^{2}+5 n-4}{2}\right] \bmod \left(2 n^{2}\right)$

$$
=\left[\frac{-n^{2}+5 n-4}{2}\right] \bmod \left(2 n^{2}\right)=\frac{3 n^{2}+5 n-4}{2},
$$

$f^{*}\left(v_{n}\right)=\left[\sum_{i=1}^{n} f\left(u_{i} v_{n}\right)\right] \bmod \left(2 n^{2}\right)=f\left(u_{n} v_{n}\right)=\frac{2 n^{2}-n+3}{2}$,

$f^{*}\left(v_{n-1}\right)=\left[\frac{-n^{3}+3 n^{2}+2 n-4}{2}\right] \bmod \left(2 n^{2}\right)=n^{2}+n-2$,

$f^{*}\left(v_{n-2}\right)=\left[\frac{n^{3}+n^{2}-2 n+8}{2}\right] \bmod \left(2 n^{2}\right)=n^{2}-n+4$,

$f^{*}\left(v_{n-3}\right)=\left[\frac{-n^{3}-n^{2}+6 n-12}{2}\right] \bmod \left(2 n^{2}\right)=n^{2}+3 n-6$,

and

$f^{*}\left(v_{n-4}\right)=\left[\frac{n^{3}+n^{2}-6 n+16}{4}\right] \bmod \left(2 n^{2}\right)=n^{2}-3 n+8$.

In the general case, we have

$f^{*}\left(v_{n-i}\right)= \begin{cases}n^{2}+i n+2 i & \text { if } i=1,3, \cdots, \frac{n-3}{2} ; \\ n^{2}-(i-1) n+2 i & \text { if } i=2,4, \cdots, \frac{n-4}{2} .\end{cases}$

Here, we notice that $f^{*}\left(v_{n-i}\right)+f^{*}\left(v_{n-(i+1)}\right)=2, i=1,2,3, \cdots, \frac{n-3}{2}$

Clearly, all the label of the vertices are even and distinct. Hence, the graph $K_{n, n}=$ $n K_{1}+n K_{1}$ has an edge even graceful labeling.

Illustration: we present an edge even graceful labeling of the graph $K_{13,13}$ in the

\begin{tabular}{|c|c|c|c|c|c|c|c|c|c|c|c|c|c|}
\hline & $v_{1}$ & $v_{2}$ & $\nu_{3}$ & $v_{4}$ & $\nu_{5}$ & $v_{6}$ & $v_{7}$ & $\nu_{8}$ & $v_{9}$ & $v_{10}$ & $v_{11}$ & $v_{12}$ & $v_{13}$ \\
\hline$u_{1}$ & 2 & 336 & 4 & 334 & 6 & 332 & 8 & 330 & 10 & 328 & 12 & 326 & 14 \\
\hline$u_{2}$ & 16 & 322 & 18 & 320 & 20 & 318 & 22 & 316 & 24 & 314 & 26 & 312 & 324 \\
\hline$u_{3}$ & 28 & 310 & 30 & 308 & 32 & 306 & 34 & 304 & 36 & 302 & 38 & 300 & 40 \\
\hline$u_{4}$ & 42 & 296 & 44 & 294 & 46 & 292 & 48 & 290 & 50 & 288 & 52 & 286 & 298 \\
\hline$u_{5}$ & 54 & 284 & 56 & 282 & 58 & 280 & 60 & 278 & 62 & 276 & 64 & 244 & 66 \\
\hline$u_{6}$ & 68 & 270 & 70 & 268 & 72 & 266 & 74 & 264 & 76 & 262 & 78 & 260 & 272 \\
\hline$u_{7}$ & 80 & 258 & 82 & 256 & 84 & 254 & 86 & 252 & 88 & 250 & 90 & 248 & 92 \\
\hline$u_{8}$ & 94 & 244 & 96 & 242 & 98 & 240 & 100 & 238 & 102 & 236 & 104 & 234 & 246 \\
\hline$u_{9}$ & 106 & 232 & 108 & 230 & 110 & 228 & 112 & 226 & 114 & 224 & 116 & 222 & 118 \\
\hline$u_{10}$ & 120 & 218 & 122 & 216 & 124 & 214 & 126 & 212 & 128 & 210 & 130 & 208 & 220 \\
\hline$u_{11}$ & 132 & 206 & 134 & 204 & 136 & 202 & 138 & 200 & 140 & 198 & 142 & 196 & 144 \\
\hline$u_{12}$ & 146 & 192 & 148 & 190 & 150 & 188 & 152 & 186 & 154 & 184 & 156 & 182 & 194 \\
\hline$u_{13}$ & 158 & 180 & 160 & 178 & 162 & 176 & 338 & 170 & 168 & 172 & 166 & 174 & $164)$ \\
\hline
\end{tabular}
following matrix 
Theorem 2 The graph $K_{m, n}=m K_{1}+n K_{1}$ has an edge even graceful labeling when $m$ and $n$ are distinct odd numbers, $m \neq 2 n-3$ and $k m \neq l n, k=1,3, \cdots, n-1, \quad l=$ $1,3, \cdots, m-1$.

Proof In the graph $K_{m, n}=m K_{1}+n K_{1}$, we have $p=\left|V\left(K_{m, n}\right)\right|=m+n$ and $q=\left|E\left(K_{m, n}\right)\right|=m n$. Without loss of generality, assume that $m>n$ and the vertices of $K_{m, n}$ divided into two disjoint sets $\left\{v_{1}, v_{2}, \cdots, v_{m}\right\}$ and $\left\{u_{1}, u_{2}, \cdots, u_{n}\right\}$. Put $v_{i}$ as the columns of the matrix and $u_{i}$ as the rows. We define the labeling function $f: E\left(K_{m, n}\right) \longrightarrow\{2,4, \cdots, 2 m n\}$ as follows: first, label the edges incident to the vertex $u_{1}$, i.e., $u_{1} v_{1}, u_{1} v_{2}, u_{1} v_{3}, u_{1} v_{4}, \cdots, u_{1} v_{n-2}, u_{1} v_{n-1}, u_{1} v_{n}$ by $2,2 m n-2,4,2 m n-4, \cdots, m-1,2 m n-(n-1), n+1$, respectively, then reverse the direction of labeling to label the edges incident to the vertex $u_{2}$ as $u_{2} v_{m}, u_{2} v_{m-1}, u_{2} v_{m-2}, u_{2} v_{m-3}, \cdots, u_{2} v_{3}, u_{2} v_{2}, u_{2} v_{1}$ by $2 m n-(n+1), m+3,2 m n-$ $(n+3), n+5, \cdots, 2 m+4,2 m n-(2 m+2), 2 m+2$ and so on.

The following matrix shows the methods of labeling

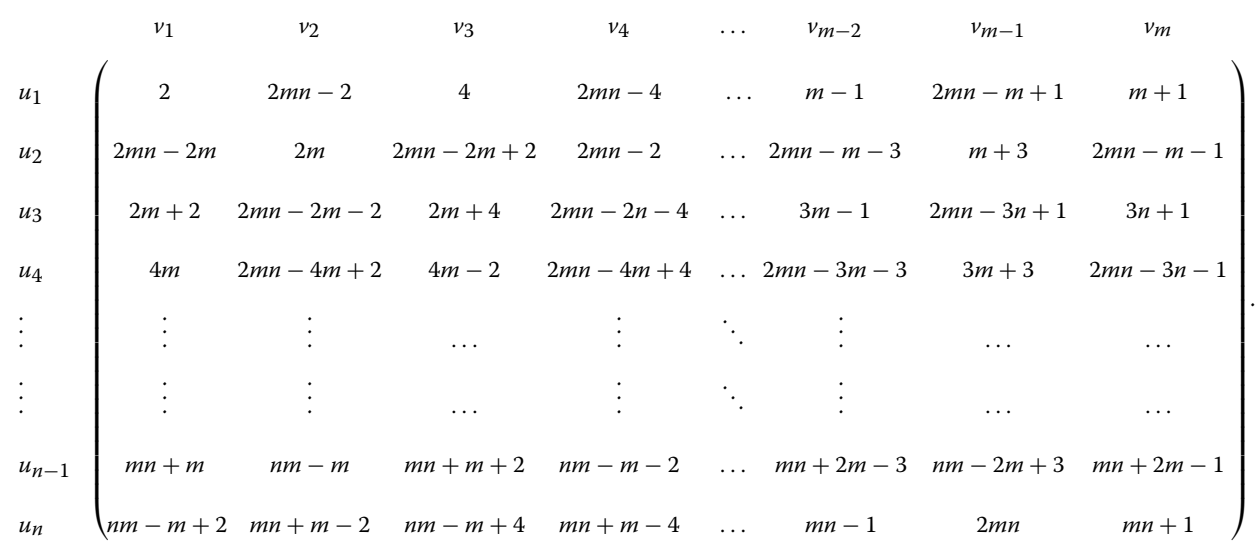

In this case, the label of the vertex $u_{n}$ will repeat with the labels of the vertex $v_{m}$. To avoid this problem we replace the labels of the two edges $u_{n} v_{m-1}$ and $u_{n} v_{m}$, that is $f\left(u_{n} v_{m-1}\right)=2 m n$ and $f\left(u_{n} v_{m}\right)=n m+1$. Thus, the induced vertex labels are

(i) The labels of the vertices $u_{i}$ is the sum of rows in the matrix, so the labels of these vertices are

$m+1,2 m n-m-1,3 m+1,2 m n-3 m-1, \cdots,(n-2) m+1, m n+2 n-1,0$, respectively.

(ii) The labels of the vertices $v_{i}, i=1,2, \cdots, m$ is the sum of columns in the matrix, we have

$$
f^{*}\left(v_{i}\right)= \begin{cases}i n+1 & \text { if } i=1,3,5 \cdots, m-2 \\ 2 m n-(i-1) n-1 & \text { if } i=2,4, \cdots, m-3 .\end{cases}
$$

Hence, the labels of the vertices $v_{1}, v_{2}, v_{3}, v_{4}, \cdots, v_{m-3}, v_{m-2}$ are $n+1,2 m n-(n+$ $1), 3 n+1,2 m n-(3 m+1), \cdots, 2 m n-[(m-4) n+1],(m-2) n+1$, respectively, and $f^{*}\left(v_{m-1}\right)=2 n-2, f^{*}\left(v_{m}\right)=n m+1$. 
Illustration: If we take $m=15$, then we can label the graphs $K_{15,7}, K_{15,11}$, and $K_{15,13}$, while we can not find labels of $K_{15,3}, K_{15,5}$, and $K_{15,9}$. We present an edge even graceful labeling of the graph $K_{15,7}=15 K_{1}+7 K_{1}$ in the following matrix

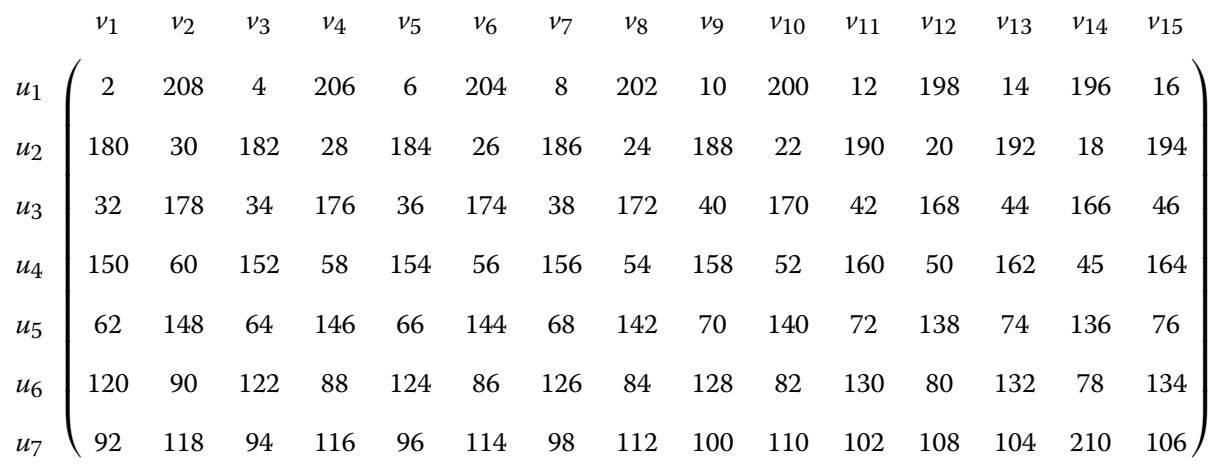

\section{Edge even graceful labeling of the join graph $\boldsymbol{k}_{\mathbf{1}}+\boldsymbol{k}_{\mathbf{1}, \boldsymbol{n}}$.}

Theorem 3 The graph $K_{1}+K_{1, n}$ has an edge even graceful labeling.

Proof Let $\left\{v_{0}, v_{1}, v_{2}, \cdots, v_{n}\right\}$ be the vertices of the graph $K_{1, n}$ with central vertex $v_{0}$ and $\{x\}$ be the vertex of $K_{1}$ so the edges of $K_{1}+K_{1, n}$ are $\left\{x v_{0}, x v_{i}, v_{0} v_{i}, i=1,2, \cdots, n\right\}$. Here, $p=\left|V\left(K_{1}+K_{1, n}\right)\right|=n+2$ and $q=\left|E\left(K_{1}+K_{1, n}\right)\right|=2 n+1$. There are two cases:

Case (1) : when $n$ is even. We define the labeling function

$$
\begin{aligned}
& f: E\left(K_{1}+K_{1, n}\right) \longrightarrow\{2,4, \cdots, 4 n+2\} \text { as follows: } \\
& f\left(x v_{0}\right)=2 n, \\
& f\left(x v_{i}\right)= \begin{cases}2 i & \text { if } i=1,2, \cdots, \frac{n}{2} ; \\
2 n+2 i & \text { if } i=\frac{n}{2}+1, \cdots, n .\end{cases}
\end{aligned}
$$

and

$$
f\left(v_{0} v_{i}\right)= \begin{cases}2 n+2 i & \text { if } i=1,2, \cdots, \frac{n}{2} \\ 4 n+2 & \text { if } i=\frac{n}{2}+1 ; \\ 2 i-2 & \text { if } i=\frac{n}{2}+2, \cdots, n .\end{cases}
$$

Therefore, the induçed vertex labels are

$$
\begin{aligned}
f^{*}(x) & =\left[f\left(x v_{0}\right)+\sum_{i=1}^{n} f\left(x v_{i}\right)\right] \bmod (4 n+2)=f\left(x v_{0}\right) \bmod (4 n+2)=2 n, \\
f^{*}\left(v_{0}\right) & =\left[f\left(x v_{0}\right)+\sum_{i=1}^{n} f\left(v_{0} v_{i}\right)\right] \bmod (4 n+2) \\
& =\left[f\left(x v_{0}\right)+f\left(a_{1}\right)\right] \bmod (4 n+2)=0, \\
f^{*}\left(v_{i}\right) & =\left[f\left(x v_{i}\right)+f\left(v_{0} v_{i}\right)\right] \bmod (4 n+2) \\
& =\left\{\begin{array}{lll}
(2 n+4 i) \bmod (4 n+2) & \text { if } \quad 1 \leq i \leq \frac{n}{2} ; \\
(2 n+4 i-2) \bmod (4 n+2) & \text { if } \quad \frac{n}{2}+2 \leq i<n .
\end{array}\right.
\end{aligned}
$$

Hence, the labels of the vertices $v_{1}, v_{2}, \cdots, v_{\frac{n}{2}-1}, v_{\frac{n}{2}}$ are $2 n+4,2 n+8, \cdots, 4 n-4,4 n$, respectively, and the labels of the vertices $v_{\frac{n}{2}+1}, v_{\frac{n}{2}+2}, v_{\frac{n}{2}+3}, \cdots, v_{n-1}, v_{n}$ are $3 n+2,4,8 \cdots, 2 n-8,2 n-4$, respectively. Finally, $f^{*}\left(v_{\frac{n}{2}+1}\right)=\left[f\left(x v_{\frac{n}{2}+1}\right)+f\left(v_{0} v_{\frac{n}{2}+1}\right)\right] \bmod (4 n+2)=3 n+2$.

Case (2): when $n$ is odd. We define the labeling function $f: E\left(K_{1}+K_{1, n}\right) \longrightarrow\{2,4, \cdots, 4 n+2\}$ as follows: 


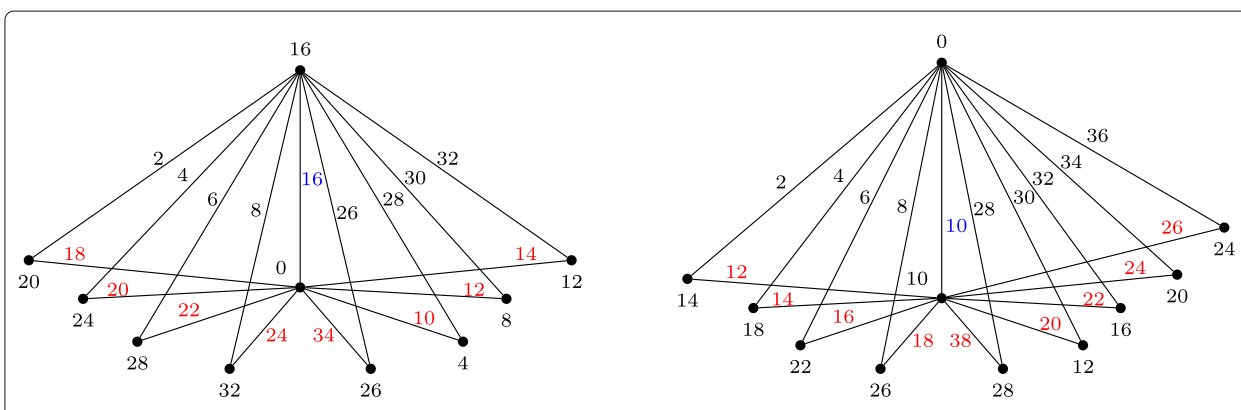

Fig. 2 An edge even graceful labeling of $K_{1}+K_{1,8}$, and $K_{1}+K_{1,9}$

$f\left(x v_{0}\right)=n+1$,

$f\left(x v_{i}\right)= \begin{cases}2 i & \text { if } i=1,2, \cdots, \frac{n-1}{2} \\ 2 n+2 i & \text { if } i=\frac{n+1}{2}, \cdots, n .\end{cases}$

and

$f\left(v_{0} v_{i}\right)= \begin{cases}n+1+2 i & \text { if } i=1,2, \ldots, \frac{n-1}{2} ; \\ 4 n+2 & \text { if } i=\frac{n+1}{2} ; \\ n-1+2 i & \text { if } i=\frac{n+3}{2}, \cdots, n .\end{cases}$

Considering the vertex labels we find

$f^{*}\left(v_{0}\right)=\left[f\left(x v_{0}\right)+\sum_{i=1}^{n} f\left(v_{0} v_{i}\right)\right] \bmod (4 n+2)=n+1, f^{*}(x)=0$ and

$$
f^{*}\left(v_{i}\right)=\left\{\begin{array}{llr}
(n+1+4 i) \bmod (4 n+2) & \text { if } & 1 \leq i \leq \frac{n-1}{2} \\
(3 n-1+4 i) \bmod (4 n+2) & \text { if } & \frac{n+3}{2} \leq i \leq n .
\end{array}\right.
$$

Then, the labels of the vertices $v_{1}, v_{2}, \cdots, v_{\frac{n-1}{2}}, v_{\frac{n+1}{2}}$ are $n+5, n+9, \cdots, 3 n-1,3 n+1$, respectively, and the labels of the vertices $v_{\frac{n+3}{2}}, v_{\frac{n+5}{2}}, \cdots, v_{n-1}, v_{n}$ are $n+3, n+7 \cdots, 3 n-7,3 n-3$, respectively.

Finally, $f^{*}\left(v_{\frac{n+1}{2}}\right)=\left[f\left(e_{\frac{n+1}{2}}\right)+f\left(a_{\frac{n+}{2}}\right)\right] \bmod (4 n+2)=3 n+1$.

Overall, all vertex labels are distinct even numbers, also $f^{*}(x)$ and $f^{*}\left(v_{0}\right)$ are different from all the labels of the vertices $v_{i}$. Hence, the graph $K_{1}+K_{1, n}$ is edge even graceful for all $n$.

Illustration: In Fig. 2, we present an edge even graceful labeling of the graphs $K_{1}+K_{1,8}$ and $K_{1}+K_{1,9}$

\section{Edge even graceful labeling of the join graph $K_{\mathbf{1}}+w_{n}$}

Theorem 4 The join graph $K_{1}+W_{n}$ has an edge even graceful labeling for all $n$.

Proof Let $\left\{v_{0}, v_{1}, v_{2}, \cdots, v_{n}\right\}$ be the vertices of $W_{n}$ with central vertex $v_{0}$ and $\{x\}$ be the vertex of $K_{1}$ so the edges of $K_{1}+W_{n}$ will be $\left\{x v_{0}, x v_{i}, v_{0} v_{i}, v_{i} v_{i+1}, i=1,2, \cdots, n\right\}$. So, $p=\left|V\left(K_{1}+W_{n}\right)\right|=n+2$ and $q=\left|E\left(K_{1}+W_{n}\right)\right|=3 n+1$. There are five cases:

Case $(1):$ For $n \equiv 0(\bmod 6)$, or $n \equiv 4(\bmod 6)$, we define the labeling function

$$
f: E\left(K_{1}+W_{n}\right) \longrightarrow\{2,4, \cdots, 6 n+2\} \text { as follows: }
$$

$$
f\left(v_{0} x\right)=6 n+2 \text {, }
$$

$$
f\left(v_{1} v_{n}\right)=3 n, \quad f\left(v_{i} v_{i+1}\right)=n+2 i \quad \text { for } i=1,2, \cdots n-1 \text {, }
$$

$f\left(x v_{i}\right)= \begin{cases}3 n+2 & \text { if } i=1 \\ 5 n-2 i+4 & \text { if } 2 \leq i \leq n .\end{cases}$

and 


$$
f\left(v_{0} v_{i}\right)= \begin{cases}2 i & \text { if } 1 \leq i \leq \frac{n}{2} \\ 4 n+2 i & \text { if } \frac{n}{2}<i \leq n\end{cases}
$$

Then, the induced vertex labels are

$$
\begin{aligned}
& f^{*}\left(v_{0}\right)=\left[\sum_{i=1}^{n} f\left(v_{0} v_{i}\right)+f\left(v_{0} x\right)\right] \bmod (6 n+2)=0 \text { and } \\
& f^{*}(x)=\left[\sum_{i=1}^{n} f\left(x v_{i}\right)+f\left(x v_{0}\right)\right] \bmod (6 n+2) \\
& \quad=\left[\sum_{i=1}^{n}(3 n+2 i)+(6 n+2)\right] \bmod (6 n+2)=\left(4 n^{2}+n\right) \bmod (6 n+2)
\end{aligned}
$$

$$
\begin{aligned}
f^{*}(x)= & {\left[4(6 k)^{2}+(6 k)\right] \bmod (36 k+2) } \\
= & {[4 k(36 k+2)-(2 k)] \bmod (36 k+2) } \\
\equiv & (-2 k) \bmod (36 k+2) \equiv(34 k+2) \bmod (36 k+2) \\
& =\left(\frac{17 n+6}{3}\right) .
\end{aligned}
$$

Similarly, if $n \equiv 4(\bmod 6) \Rightarrow n=6 k+4 \Rightarrow 2 q=6 n+2=36 k+26$, then, $f^{*}(x)=\left[4(6 k+4)^{2}+(6 k+4)\right] \bmod (36 k+26)=\left(\frac{11 n+4}{3}\right)$.

Also, $f^{*}\left(v_{1}\right)=\left[f\left(v_{1} v_{2}\right)+f\left(v_{n} v_{1}\right)+f\left(x v_{1}\right)+f\left(v_{0} v_{1}\right)\right] \bmod (6 n+2)=n+4$ and $f^{*}\left(v_{i}\right)=\left[f\left(v_{i} v_{i+1}\right)+f\left(v_{i-1} v_{i}\right)+f\left(x v_{i}\right)+f\left(v_{0} v_{i}\right)\right] \bmod (6 n+2)$

$$
=\left\{\begin{array}{lll}
(n+4 i) \bmod (6 n+2) & \text { if } \quad 2 \leq i \leq \frac{n}{2} \\
(5 n+4 i) \bmod (6 n+2) & \text { if } \quad \frac{n}{2}+1 \leq i \leq n .
\end{array}\right.
$$

Hence, the labels of the vertices $v_{1}, v_{2}, v_{3}, \cdots, v_{\frac{n}{2}}$ will be

$n+4, n+8, n+12, \cdots, 3 n$, respectively, and the labels of the vertices

$v_{\frac{n}{2}+1}, v_{\frac{n}{2}+2}, \cdots, v_{n-1}, v_{n}$ will be $n+2, n+6, \cdots, 3 n-6,3 n-2$,

respectively, which are all even and distinct numbers.

Case (2): For $n \equiv 2(\bmod 6)$, we define the labeling function $f$ as follows:

$f\left(x v_{0}\right)=6 n+2$,

$f\left(x v_{i}\right)= \begin{cases}2 i & \text { if } 1 \leq i \leq \frac{n}{2} \\ 4 n+2 i & \text { if } \frac{n}{2}<i \leq n,\end{cases}$

$f\left(v_{i} v_{i+1}\right)= \begin{cases}3 n+2 i & \text { if } 1 \leq i \leq n-1 ; \\ n+2 & \text { if } i=n,\end{cases}$

and

$f\left(v_{0} v_{i}\right)= \begin{cases}5 n & \text { if } i=1 ; \\ 3 n+4-2 i & \text { if } 2 \leq i \leq n .\end{cases}$

In view of the above labeling patten and since

$n \equiv 2(\bmod 6) \Rightarrow n=6 k+2 \Rightarrow 2 q=6 n+2=36 k+14$ then the induced vertex labels are

$$
\begin{gathered}
f^{*}\left(v_{0}\right)=\left(2 n^{2}+5 n-2\right) \bmod (6 n+2)= \\
{[2 k(36 k+14)+(94 k+68)] \bmod (36 k+14)} \\
\equiv(14 k+2) \bmod (36 k+14)=\left(\frac{7 n-8}{3}\right) .
\end{gathered}
$$

By the same way, in the first case, we have

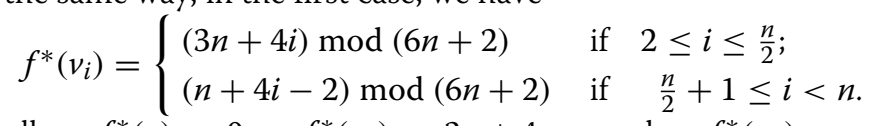

Finally, $f^{*}(x)=0, \quad f^{*}\left(v_{1}\right)=3 n+4 \quad$ and $f^{*}\left(v_{n}\right)=n$.

Therefore, the labels of the vertices $v_{1}, v_{2}, v_{3}, \cdots, v_{\frac{n}{2}}$ are $3 n+4,3 n+8,3 n+12, \cdots, 5 n, \quad$ respectively, and the labels of the vertices $v_{\frac{n}{2}+1}, v_{\frac{n}{2}+2}, \cdots, v_{n-1}, v_{n}$ are $3 n+2,3 n+6, \cdots, 5 n-6, n$, respectively. 
Case (3): For $n \equiv 3(\bmod 6)$, we define the labeling $f: E\left(K_{1}+W_{n}\right) \rightarrow\{2,4, \cdots, 6 n+2\}$ as follows:

$$
f\left(x v_{0}\right)=6 n, \quad f\left(x v_{i}\right)=3 n+3-2 i \quad \text { for } i=1,2, \cdots n,
$$

$f\left(v_{i} v_{i+1}\right)= \begin{cases}3 n+1+2 i & \text { if } i=1,2, \cdots, n-1 \\ 6 n+2 & \text { if } i=n\end{cases}$

and

$$
f\left(v_{0} v_{i}\right)= \begin{cases}2 i & \text { if } 1 \leq i \leq \frac{n+1}{2} \\ 4 n+2 i-2 & \text { if } \frac{n+3}{2} \leq i \leq n\end{cases}
$$

In this labeling, the induced vertex labels are

$$
\begin{aligned}
& f^{*}\left(v_{0}\right)=\left[\sum_{i=1}^{n} f\left(v_{0} v_{i}\right)+f\left(x v_{0}\right)\right] \bmod (6 n+2)=0, \\
& f^{*}\left(v_{1}\right)=\left[f\left(v_{1} v_{2}\right)+f\left(v_{n} v_{1}\right)+f\left(v_{0} v_{1}\right)+f\left(x v_{1}\right)\right] \bmod (6 n+2)=4, \\
& f^{*}(x)=\left[\sum_{i=1}^{n} f\left(x v_{i}\right)+f\left(v_{0} x\right)\right] \bmod (6 n+2)=\left(2 n^{2}+2 n-2\right) \bmod (6 n+2) . \\
& \text { Since } n \equiv 3(\bmod 6) \Rightarrow n=6 k+3 \Rightarrow 2 q=6 n+2=36 k+20 . \\
& \text { Then, } f^{*}(x)=\left(\frac{4 n-6}{3}\right) . \\
& \text { And } f^{*}\left(v_{i}\right)=\left[f\left(v_{i} v_{i+1}\right)+f\left(v_{i-1} v_{i}\right)+f\left(v_{0} v_{i}\right)+f\left(x v_{i}\right)\right] \bmod (6 n+2) \\
& \quad=\left\{\begin{array}{lll}
(3 n+4 i+1) \bmod (6 n+2) & \text { if } \quad 2 \leq i \leq \frac{n+1}{2} \\
(n+4 i-3) \bmod (6 n+2) & \text { if } \quad \frac{n+3}{2} \leq i \leq n-1 .
\end{array}\right.
\end{aligned}
$$

Finally,

$f^{*}\left(v_{n}\right)=\left[f\left(v_{n-1} v_{n}\right)+f\left(v_{n} v_{1}\right)+f\left(v_{0} v_{n}\right)+f\left(x v_{n}\right)\right] \bmod (6 n+2)=6 n-2$.

Hence, the labels of the vertices $v_{1}, v_{2}, v_{3}, \cdots, v_{\frac{n-1}{2}}, v_{\frac{n+1}{2}}$ are

$4,3 n+9,3 n+13, \cdots, 5 n-1,5 n+3$, respectively, and the labels of the vertices $v_{\frac{n+3}{2}}, v_{\frac{n+5}{2}}, v_{\frac{n+7}{2}}, \cdots, v_{n-1}, v_{n}$ are $3 n+3,3 n+7,3 n+11, \cdots, 5 n-7,6 n-2$, respectively. There is no repetition in the vertex label, also $f^{*}(x)$ and $f^{*}\left(v_{0}\right)$ are different from all the labels of the vertices $v_{i}$.

Case (4) : For $n \equiv 5(\bmod 6), n>5$, we define the labeling function $f$ as follows:

$$
\begin{aligned}
& f\left(x v_{0}\right)=2, \quad f\left(x v_{i}\right)=(3 n+3)-2 i \quad \text { for } i=1,2, \cdots n, \\
& f\left(v_{i} v_{i+1}\right)= \begin{cases}3 n+1+2 i & \text { if } i=1,2, \cdots, n-1 \\
6 n+2 & \text { if } i=n,\end{cases}
\end{aligned}
$$

and

$$
f\left(v_{0} v_{i}\right)= \begin{cases}2 i+2 & \text { if } 1 \leq i \leq \frac{n-1}{2} \\ 4 n+2 i & \text { if } \frac{n+1}{2} \leq i \leq n\end{cases}
$$

In view of the above labeling pattern, the induced vertex labels are

Since $n \equiv 5(\bmod 6) \Rightarrow n=6 k+5 \Rightarrow 2 q=6 n+2=36 k+30$, then $f^{*}(x)=\left(2 n^{2}+2 n+2\right) \bmod (6 n+2)=\left(\frac{16 n+10}{3}\right) \bmod (6 n+2)$.

Also, $f^{*}\left(v_{i}\right)= \begin{cases}(3 n+4 i+3) \bmod (6 n+2) & \text { if } 2 \leq i \leq \frac{n-1}{2} \\ (n+4 i-1) \bmod (6 n+2) & \text { if } \quad \frac{n+1}{2} \leq i<n .\end{cases}$

Finally, $f^{*}\left(v_{0}\right)=0, \quad f^{*}\left(v_{1}\right)=6$ and $f^{*}\left(v_{n}\right)=6 n$.

Hence, the labels of the vertices $v_{1}, v_{2}, v_{3}, \cdots, v_{\frac{n-3}{2}}, v_{\frac{n-1}{2}}$ are

$6,3 n+11,3 n+15, \cdots, 5 n-3,5 n+1$, respectively, and the labels of the vertices $v_{\frac{n+1}{2}}, v_{\frac{n+3}{2}}, \cdots, v_{n-1}, v_{n}$ are $3 n+1,3 n+5, \cdots, 5 n-5,6 n$, respectively. Clearly, $f^{*}(x)$ and $f^{*}\left(\nu_{0}\right)$ are different from all the labels of the vertices $v_{i}$.

When $n=5$, the graph $E\left(K_{1}+W_{5}\right)$ is an edge even graceful graph but it does not follow this rule, see Fig. 4.

Case (5): For $n \equiv 1(\bmod 6)$, we defined the labeling function $f$ as follows: $f\left(v_{0} x\right)=6 n$

$$
f\left(v_{1} v_{n}\right)=4 n, \quad f\left(v_{i} v_{i+1}\right)=2 n+2 i \quad \text { for } i=1,2, \cdots n-1,
$$




$$
f\left(x v_{i}\right)= \begin{cases}2 i & \text { if } 1 \leq i \leq \frac{n+1}{2} \\ 4 n-2+2 i & \text { if } \quad \frac{n+3}{2} \leq i \leq n\end{cases}
$$

and

$$
f\left(v_{0} v_{i}\right)= \begin{cases}5 n+1-2 i & \text { if } 1 \leq i \leq \frac{n-1}{2} \\ 6 n+2 & \text { if } i=\frac{n+1}{2} \\ 3 n+3-2 i & \text { if } \frac{n+3}{2} \leq i \leq n\end{cases}
$$

In this case, the induced vertex labels are

$$
\begin{aligned}
& f^{*}(x)=0, \quad f^{*}\left(v_{0}\right)=6 n, \quad f^{*}\left(v_{1}\right)=5 n+1, \\
& \text { and } \\
& f^{*}\left(v_{i}\right)= \begin{cases}(3 n+4 i-3) \bmod (6 n+2) & \text { if } 2 \leq i \leq \frac{n-1}{2} \\
(5 n+4 i-3) \bmod (6 n+2) \equiv(4 i-n-5) & \text { if } \frac{n+3}{2} \leq i \leq n\end{cases}
\end{aligned}
$$

Finally,

$$
f^{*}\left(v_{\frac{n+1}{2}}\right)=\left[f\left(v_{\frac{n+1}{2}} v_{\frac{n+3}{2}}\right)+f\left(v_{\frac{n-1}{2}} v_{\frac{n+1}{2}}\right)+f\left(v_{0} v_{\frac{n+1}{2}}\right)+f\left(x v_{\frac{n+1}{2}}\right)\right] \bmod (6 n+2)=n-1 .
$$

It is clear that for all $i \in\{1,2,3, \ldots, n\}$, the labels of the vertices $v_{i}$ are all distinct, even and different from $f^{*}(x)$ and $f^{*}\left(v_{0}\right)$ which complete the proof.

Illustration: In Fig. 3, we present an edge even graceful labeling of the graphs $K_{1}+W_{8}$ and $K_{1}+W_{10}$.

Illustration: In Fig. 4, we present an edge even graceful labeling of the graphs $K_{1}+W_{9}$ $, K_{1}+W_{11}, K_{1}+W_{5}$ and $K_{1}+W_{7}$.

\section{Edge even graceful labeling of the join graph $K_{\mathbf{1}}+\boldsymbol{s f _ { \boldsymbol { n } }}$}

The sunflower graph, $s f_{n}$, is defined as a graph obtained by starting with an $n$-cycle $C_{n}$ with a consecutive vertices $v_{1}, v_{2}, \cdots, v_{n}$ and creating new vertices $u_{1}, u_{2}, \cdots, u_{n}$, with $u_{i}$ connected to $v_{i}$ and $v_{i+1}$. The graph $s f_{n}$ has number of vertices $p=2 n$ and a number of edges $q=3 n$.

Theorem 5 The join graph $K_{1}+s f_{n}$ has an edge even graceful labeling for all $n$.

Proof Let $\{x\}$ be the vertex of $K_{1}$ and the edges of the graph $K_{1}+s f_{n}$ will be

$\left\{x v_{i}, x u_{i}, v_{i} v_{i+1}, v_{i} u_{i}, v_{i+1} u_{i}, \quad i=1,2, \cdots, n\right\}$. Let us use the standard notation $p=\left|V\left(K_{1}+s f_{n}\right)\right|=2 n+1$ and $q=\left|E\left(K_{1}+s f_{n}\right)\right|=5 n$.

We define the labeling function $f: E\left(K_{1}+s f_{n}\right) \longrightarrow\{2,4, \cdots, 10 n\}$ as follows:

for $i=1,2, \cdots, n, \quad f\left(x v_{i}\right)=2 i, \quad f\left(x u_{i}\right)=10 n-2 i$,

for $i=1,2, \cdots, n, \quad f\left(v_{i} u_{i}\right)=4 n+2 i, \quad f\left(u_{i} v_{i+1}\right)=2 n+2 i$,
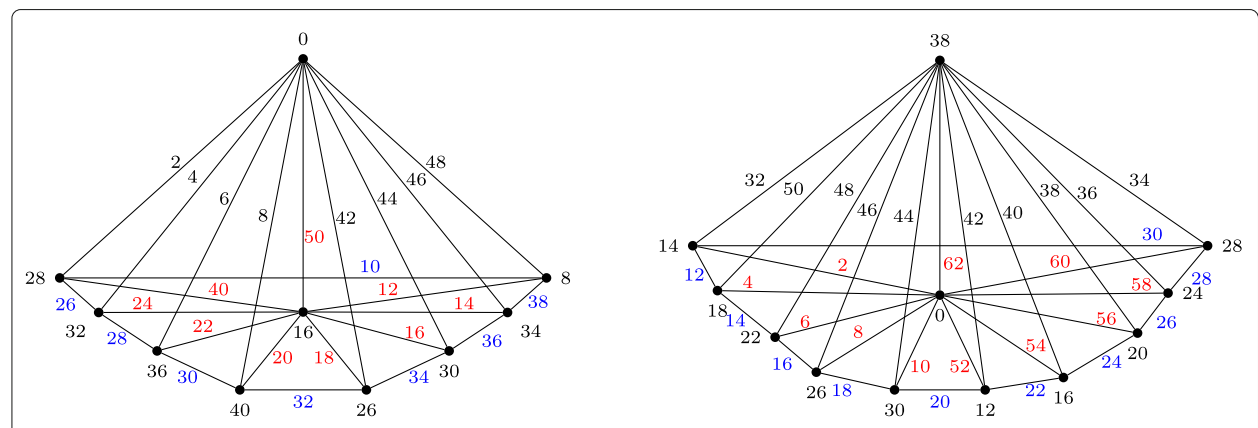

Fig. 3 An edge even graceful labeling of the graphs $K_{1}+W_{8}$ and $K_{1}+W_{10}$ 


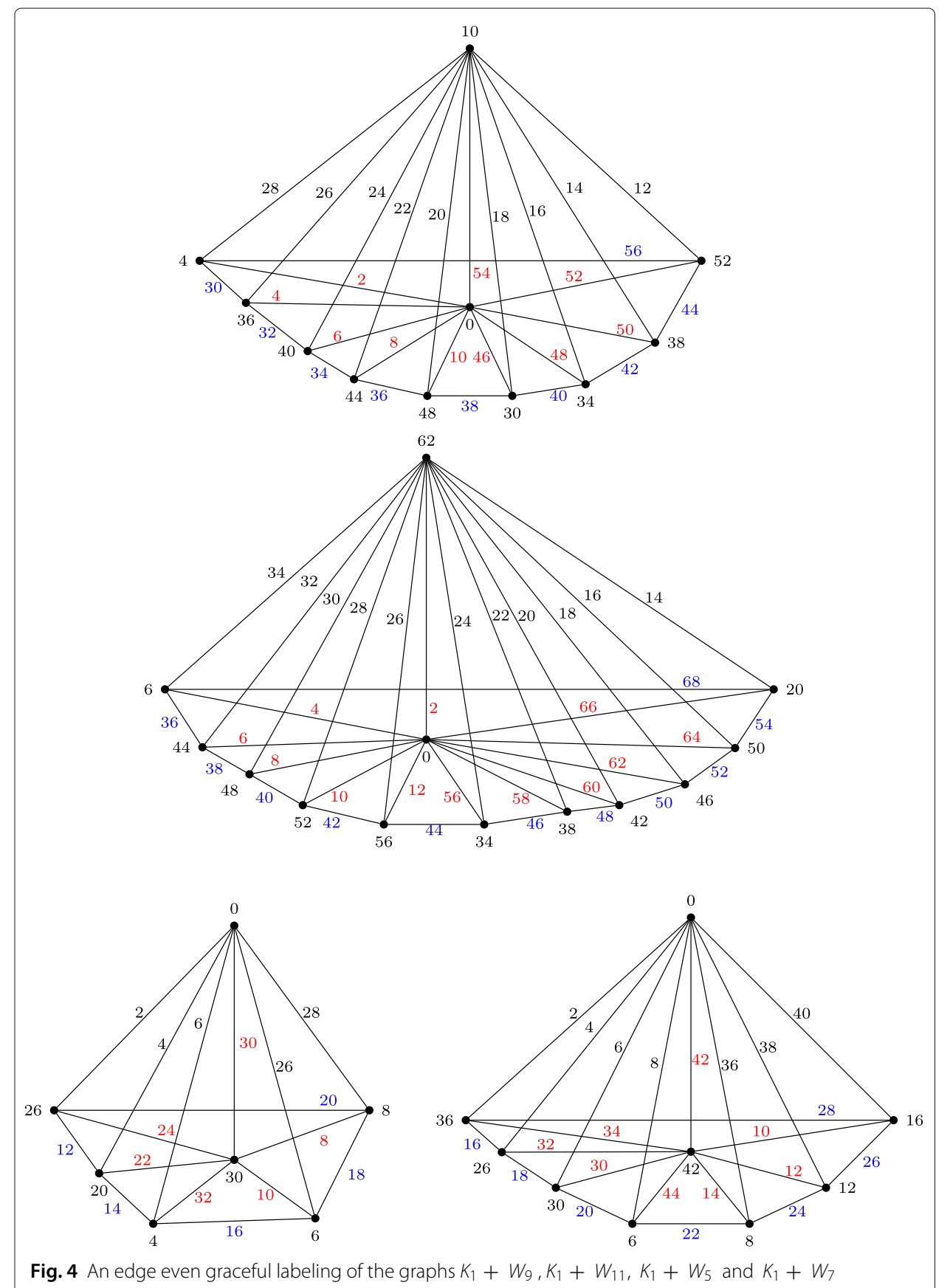

and

$f\left(v_{1} v_{n}\right)=6 n+2, \quad f\left(v_{i} v_{i+1}\right)= \begin{cases}10 n & \text { if } i=1 ; \\ 8 n+2-2 i & \text { if } i=2,3, \cdots, n-1 .\end{cases}$

Considering the given vertex labels, the induced vertex labels are

$$
\begin{aligned}
& f^{*}(x)=\left[\sum_{i=1}^{n} f\left(x v_{i}\right)+\sum_{i=1}^{n} f\left(x u_{i}\right)\right] \bmod (10 n)=0, \\
& f^{*}\left(v_{1}\right)=4 n+6, \quad f^{*}\left(v_{2}\right)=4 n+8, \\
& f^{*}\left(v_{i}\right)=2 n+2 i+4 \quad \text { for } i=3,4, \cdots, n,
\end{aligned}
$$

and

$$
f^{*}\left(u_{i}\right)=6 n+2 i \quad \text { for } i=1,2, \cdots, n .
$$




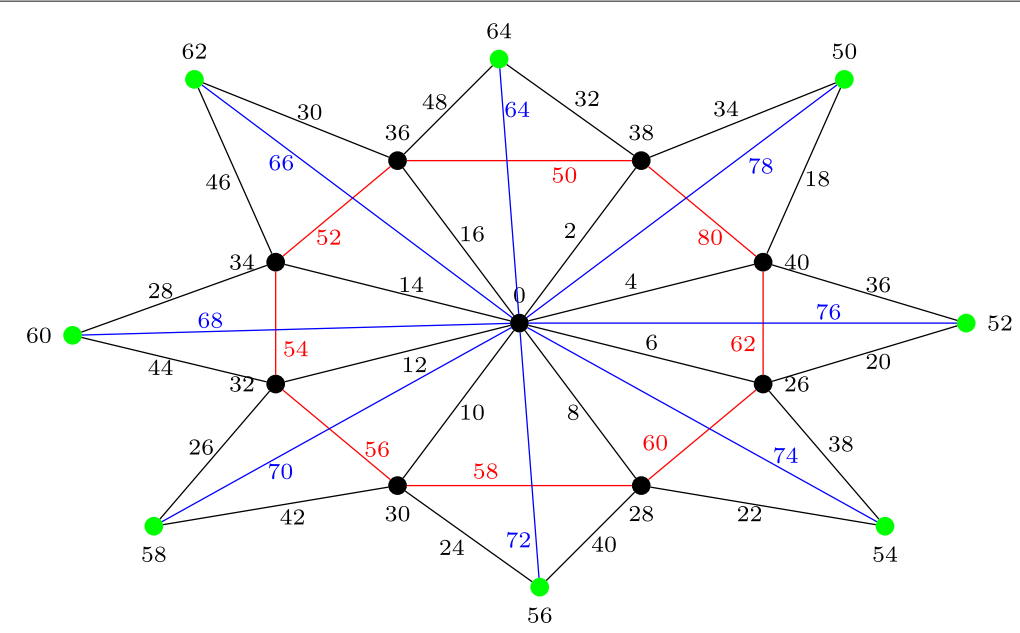

Fig. 5 An edge even graceful labeling of the graph $K_{1}+s f_{8}$

Thus, the labels of the vertices $v_{3}, v_{4}, \cdots, v_{n}$ are $2 n+10,2 n+12, \cdots, 4 n+4$, respectively, and the labels of the vertices $u_{1}, u_{2}, \cdots, u_{n}$ are $6 n+2,6 n+4, \cdots, 8 n$, respectively. Clearly, all the labels are even and distinct. Thus, the graph $K_{1}+s f_{n}$ is an edge even graceful labeling.

Illustration: In Fig. 5, we present an edge even graceful labeling of the graph $K_{1}+s f_{8}$.

\section{Edge even graceful labeling of the join graph $\overline{\boldsymbol{K}}_{\mathbf{2}}+\boldsymbol{K}_{\mathbf{1 , n}}$}

Theorem 6 The graph $\bar{K}_{2}+K_{1, n}$ has an edge even graceful labeling for all $n$.

Proof Let $\left\{v_{0}, v_{1}, v_{2}, \cdots, v_{n}\right\}$ be the vertices of the graph $K_{1, n}$ with central vertex $v_{0}$ and $\{x, y\}$ be the vertices of $\bar{K}_{2}$ so the edges of $\bar{K}_{2}+K_{1, n}$ are $\left\{x v_{0}, x v_{i}, v_{0} v_{i}, y v_{0}, y v_{i}, i=\right.$ $1,2, \cdots, n\}$. Let us use the standard notation $p=\left|V\left(\bar{K}_{2}+K_{1, n}\right)\right|=n+3$ and $q=$ $\left|E\left(\bar{K}_{1}+K_{1, n}\right)\right|=3 n+2$. There are two cases:

Case (1): when $n$ is even. We define the labeling function

$$
\begin{aligned}
& f: E\left(\bar{K}_{2}+K_{1, n}\right) \longrightarrow\{2,4, \cdots, 6 n+4\} \text { as follows: } \\
& f\left(x v_{i}\right)= \begin{cases}6 n+4 & \text { if } i=0 ; \\
2 i & \text { if } 1 \leq i \leq \frac{n}{2} \\
4 n+2+2 i & \text { if } \frac{n}{2}+1 \leq i \leq n,\end{cases} \\
& f\left(y v_{i}\right)= \begin{cases}3 n+4 & \text { if } i=0 ; \\
n+2 i & \text { if } 1 \leq i \leq \frac{n}{2} ; \\
3 n+2+2 i & \text { if } \frac{n}{2}+1 \leq i \leq n,\end{cases}
\end{aligned}
$$

and

$$
f\left(v_{0} v_{i}\right)= \begin{cases}2 n+2 i & \text { if } \quad 1 \leq i \leq \frac{n}{2}+1 \\ 2 n+2+2 i & \text { if } \quad \frac{n}{2}+2 \leq i \leq n .\end{cases}
$$

Considering the given vertex labels, the induced vertex labels are

$$
\begin{gathered}
f^{*}(x)=\left[f\left(x v_{0}\right)+\sum_{i=1}^{n} f\left(x v_{i}\right)\right] \bmod (6 n+4)=f\left(x v_{0}\right) \bmod (6 n+4)=0, \\
f^{*}(y)=\left[f\left(y v_{0}\right)+\sum_{i=1}^{n} f\left(y v_{i}\right)\right] \bmod (6 n+4)=f\left(y v_{0}\right) \bmod (6 n+4)=3 n+4, \\
f^{*}\left(v_{0}\right)=\left[\sum_{i=1}^{n} f\left(v_{0} v_{i}\right)+f\left(x v_{0}\right)+f\left(y v_{0}\right)\right] \bmod (6 n+4)=3 n+2, \\
f^{*}\left(v_{i}\right)=\left[f\left(v_{0} v_{i}\right)+f\left(x v_{i}\right)+f\left(y v_{i}\right)\right] \bmod (6 n+4)
\end{gathered}
$$


and

$$
=\left\{\begin{array}{llc}
(3 n+6 i) \bmod (6 n+4) & \text { if } & 2 \leq i \leq \frac{n}{2} \\
(3 n+2+6 i) \bmod (6 n+4) & \text { if } & \frac{n}{2}+2 \leq i \leq n .
\end{array}\right.
$$

$f^{*}\left(v_{\frac{n}{2}+1}\right)=\left[f\left(v_{0} v_{\frac{n}{2}+1}\right)+f\left(x v_{\frac{n}{2}+1}\right)+f\left(y v_{\frac{n}{2}+1}\right)\right] \bmod (6 n+4)=2$.

Thus, the labels of the vertices $v_{1}, v_{2}, \cdots, v_{\frac{n}{2}}-1, v_{\frac{n}{2}}$ are

$3 n+6,3 n+12, \cdots, 6 n-6,6 n, \quad$ respectively, and the labels of the vertices $v_{\frac{n}{2}+1}, v_{\frac{n}{2}+2}, v_{\frac{n}{2}+3}, \cdots, v_{n-1}, v_{n}$ are

$2,10,16, \cdots, 3 n-8,3 n-2$, respectively. Clearly, $f^{*}(x), f^{*}(y)$ and $f^{*}\left(v_{0}\right)$ are different from all the labels of the vertices $v_{i}$.

Case (2): when $n$ is odd. We introduce two different labeling

Method 1: We define the labeling function $f$ as follows:

and

$$
\begin{aligned}
& f\left(v_{0} v_{i}\right)= \begin{cases}2 n+2+2 i & \text { if } 1 \leq i \leq \frac{n-1}{2} \\
5 n+1-2 i & \text { if } \frac{n+1}{2} \leq i \leq n-1 ; \\
6 n+2 & \text { if } i=n,\end{cases} \\
& f\left(x v_{i}\right)= \begin{cases}4 & \text { if } i=0 \\
4+2 i & \text { if } 1 \leq i \leq \frac{n-1}{2} \\
4 n+2 i & \text { if } \frac{n+1}{2} \leq i \leq n,\end{cases}
\end{aligned}
$$

$$
f\left(y v_{i}\right)= \begin{cases}2 & \text { if } i=0 ; \\ 5 n+1-2 i & \text { if } 1 \leq i \leq \frac{n-1}{2} \\ 3 n+3-2 i & \text { if } \frac{n+1}{2} \leq i \leq n-1 ; \\ 6 n+4 & \text { if } i=n .\end{cases}
$$

Hence, the induced vertex labels are

$$
\begin{aligned}
& f^{*}(x)=\left[f\left(x v_{0}\right)+\sum_{i=1}^{n} f\left(x v_{i}\right)\right] \bmod (6 n+4)=f\left(x v_{0}\right) \bmod (6 n+4)=0 \text {, } \\
& f^{*}(y)=\left[f\left(y v_{0}\right)+\sum_{i=1}^{n} f\left(y v_{i}\right)\right] \bmod (6 n+4)=f\left(y v_{0}\right) \bmod (6 n+4)=2 \text {, } \\
& f^{*}\left(v_{0}\right)=\left[\sum_{i=1}^{n} f\left(v_{0} v_{i}\right)+f\left(x v_{0}\right)+f\left(y v_{0}\right)\right] \bmod (6 n+4)=4, \\
& f^{*}\left(v_{i}\right)= \begin{cases}(n+3+2 i) \bmod (6 n+4) & \text { if } \quad 1 \leq i \leq \frac{n-1}{2} \\
(6 n-2 i) \bmod (6 n+4) & \text { if } \quad \frac{n+1}{2} \leq i \leq n-1 .\end{cases}
\end{aligned}
$$

and $f^{*}\left(v_{n}\right)=\left[f\left(v_{0} v_{n}\right)+f\left(x v_{n}\right)+f\left(y v_{n}\right)\right] \bmod (6 n+4)=6 n-2$.

Then the labels of the vertices $v_{1}, v_{2}, \cdots, v_{\frac{n-3}{2}}, v_{\frac{n-1}{2}}$ are $n+5, n+7, \cdots, 2 n, 2 n+2$, respectively, and the labels of the vertices $v_{\frac{n+1}{2}}, v_{\frac{n+3}{2}}, \cdots, v_{n-2}, v_{n-1}, v_{n}$ are $5 n-1,5 n-$ $3, \cdots, 4 n+4,4 n+2,6 n-2$, respectively. Clearly $f^{*}(x), f^{*}(y)$ and $f^{*}\left(v_{0}\right)$ are different from all the labels of the vertices $v_{i}$. Thus the graph $\bar{K}_{2}+K_{1, n}$ has an edge even graceful labeling for all $n$.

Method 2: We can find another labeling when $n$ is an odd number, by redefining the labeling function as follows:

$$
\begin{aligned}
& f\left(x v_{i}\right)= \begin{cases}5 n+3 & \text { if } i=0 \\
2 i & \text { if } 1 \leq i \leq \frac{n+1}{2} \\
4 n+2 i+2 & \text { if } \frac{n+3}{2} \leq i \leq n,\end{cases} \\
& f\left(y v_{i}\right)= \begin{cases}6 n+4 & \text { if } i=0 \\
n+1+2 i & \text { if } 1 \leq i \leq \frac{n+1}{2} \\
3 n+1+2 i & \text { if } \frac{n+3}{2} \leq i \leq n\end{cases}
\end{aligned}
$$


and

$$
f\left(v_{0} v_{i}\right)=\left\{\begin{array}{llc}
2 n+2+2 i & \text { if } & 1 \leq i \leq \frac{n+1}{2} \\
5 n+5-2 i & \text { if } & \frac{n+3}{2} \leq i \leq n .
\end{array}\right.
$$

Then, by the same way, we can calculate $f^{*}(x), f^{*}(y)$ and $f^{*}\left(v_{0}\right)$ and prove that they are different from all the labels of the vertices $v_{i}$.

Illustration: In Fig. 6, we present an edge even graceful labeling of $\bar{K}_{2}+K_{1,10}$ and $\bar{K}_{2}+K_{1,11}$.

\section{Edge even graceful labeling of the join graph $\bar{K}_{\mathbf{2}}+w_{n}$}

Theorem 7 The graph $\bar{K}_{2}+W_{n}$ has an edge even graceful labeling for all $n$.

Proof Let $\left\{v_{0}, v_{1}, v_{2}, \cdots, v_{n}\right\}$ be the vertices of the wheel graph $W_{n}$ with central vertex $v_{0}$ and $\{x, y\}$ be the vertices of the graph $\bar{K}_{2}$, so $E\left(\bar{K}_{2}+W_{n}\right)=$ $\left\{x v_{0}, x v_{i}, y v_{0}, y v_{i}, v_{0} v_{i}, v_{i} v_{i+1}, i=1,2, \cdots, n\right\}$. In this graph, $p=\left|V\left(\bar{K}_{2}+w_{n}\right)\right|=$ $n+3$ and $q=\left|E\left(\bar{K}_{2}+W_{n}\right)\right|=4 n+2$. There are two cases:

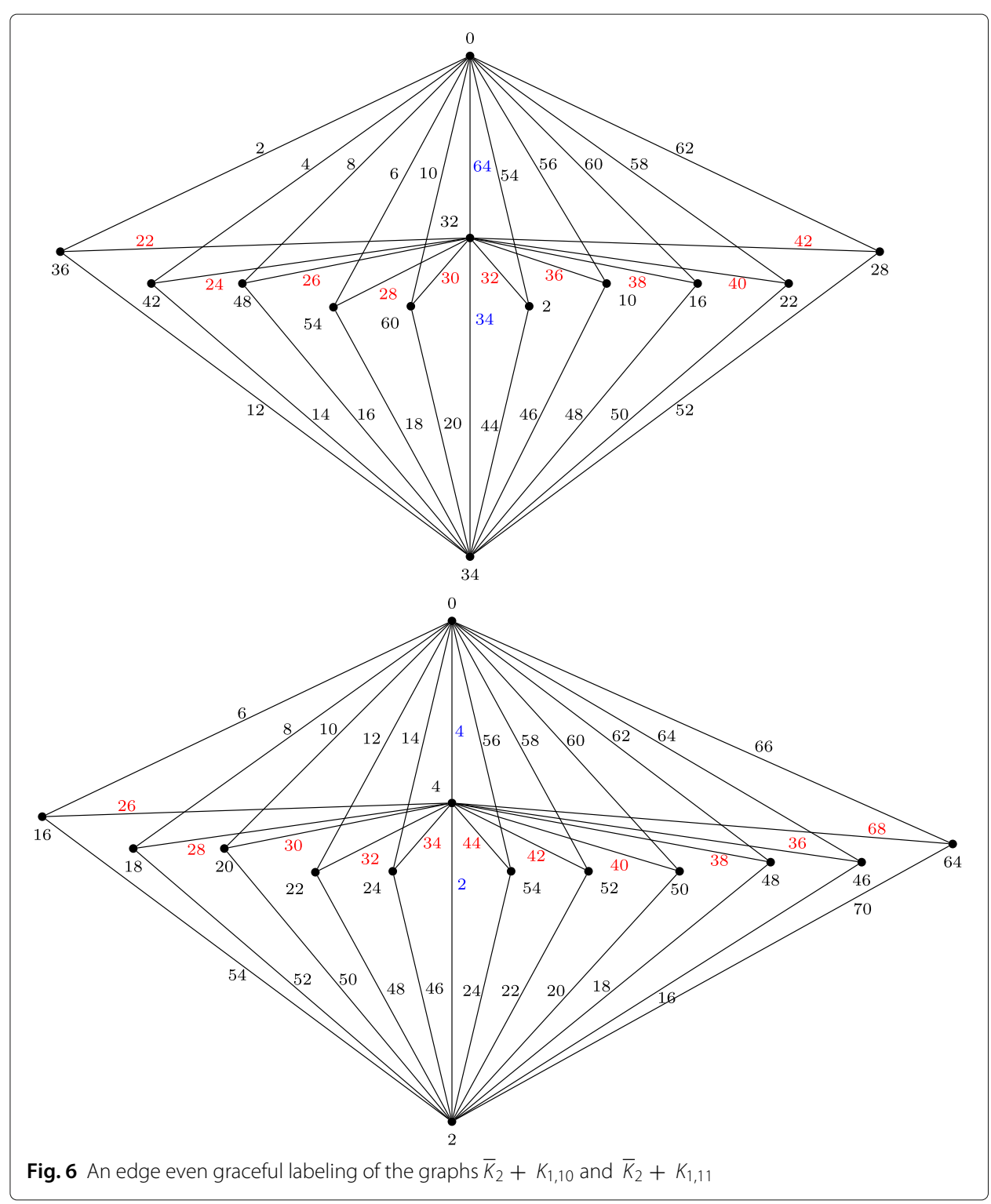


Case (1): when $n$ is even, we define the labeling $f: E\left(\bar{K}_{2}+W_{n}\right) \longrightarrow\{2,4, \cdots, 8 n+4\}$ as follows:

$$
\begin{aligned}
& f\left(v_{1} v_{n}\right)=8 n+4, \quad f\left(v_{i} v_{i+1}\right)=3 n+2 i+2 \\
& f\left(v_{0} v_{i}\right)= \begin{cases}2 i+2 & \text { if } 1 \leq i \leq \frac{n}{2} ; \\
6 n+2 i & \text { if } \frac{n}{2}<i \leq n,\end{cases} \\
& f\left(x v_{i}\right)= \begin{cases}2 & \text { if } i=0 ; \\
n+2+2 i & \text { if } 1 \leq i \leq \frac{n}{2} ; \\
5 n+2 i & \text { if } \frac{n}{2}<i \leq n,\end{cases}
\end{aligned}
$$

and

$$
f\left(y v_{i}\right)= \begin{cases}8 n & \text { if } i=0 \\ 2 n+2+2 i & \text { if } 1 \leq i \leq \frac{n}{2} \\ 4 n+2 i & \text { if } \frac{n}{2}<i \leq n\end{cases}
$$

In view of the above labeling pattern we have

$f^{*}\left(v_{1}\right)=6 n+16, f^{*}\left(v_{n}\right)=2 n-12$,

$f^{*}(x)=\left[f\left(x v_{0}\right)+\sum_{i=1}^{n} f\left(x v_{i}\right)\right] \bmod (8 n+4)=f\left(x v_{0}\right) \bmod (8 n+4)=2$,

$f^{*}(y)=\left[f\left(y v_{0}\right)+\sum_{i=1}^{n} f\left(y v_{i}\right)\right] \bmod (8 n+4)=f\left(y v_{0}\right) \bmod (8 n+4)=8 n+2$,

$f^{*}\left(v_{0}\right)=\left[\sum_{i=1}^{n} f\left(v_{0} v_{i}\right)+f\left(x v_{0}\right)+f\left(y v_{0}\right)\right] \bmod (8 n+4)=0$,

and $f^{*}\left(v_{i}\right)=\left[f\left(v_{i} v_{i+1}\right)+f\left(v_{i-1} v_{i}\right)+f\left(v_{0} v_{i}\right)+f\left(x v_{i}\right)+f\left(y v_{i}\right)\right] \bmod (8 n+4)$.

Therefore, $\quad f^{*}\left(v_{i}\right)=\left\{\begin{array}{lll}(n+4+10 i) \bmod (8 n+4) & \text { if } & 2 \leq i \leq \frac{n}{2} \\ (5 n-6+10 i) \bmod (8 n+4) & \text { if } & \frac{n}{2}+1 \leq i<n .\end{array}\right.$

Hence, the labels of the vertices $v_{2}, v_{3}, v_{4}, \cdots, v_{\frac{n}{2}}$ are

$n+24, n+34, n+44, \cdots, 6 n+4$, respectively, and the labels of the vertices

$v_{\frac{n}{2}+1}, v_{\frac{n}{2}+2}, \cdots, v_{n-1}, v_{n}$ are $2 n, 2 n+10, \cdots, 7 n-20$, respectively, which

are even and distinct numbers. Clearly, $f^{*}(x)$ and $f^{*}\left(v_{0}\right)$ are even and different from all the labels of the vertices $v_{i}$.

Case (2): when $n$ is odd, we define the labeling function $f$ as follows:

$$
\begin{aligned}
& f\left(v_{1} v_{n}\right)=5 n+1, \quad f\left(v_{i} v_{i+1}\right)=5 n-2 i+1 \quad \text { for } i=1,2, \cdots n-1 \text {, } \\
& f\left(v_{0} v_{i}\right)= \begin{cases}n+2 i+1 & \text { if } 1 \leq i \leq \frac{n+1}{2} \\
5 n-1+2 i & \text { if } \quad \frac{n+3}{2} \leq i \leq n,\end{cases} \\
& f\left(x v_{i}\right)= \begin{cases}n+1 & \text { if } i=0 \\
2 i & \text { if } 1 \leq i \leq \frac{n-1}{2} \\
6 n+2+2 i & \text { if } \frac{n+1}{2} \leq i \leq n\end{cases}
\end{aligned}
$$

and

$$
f\left(y v_{i}\right)= \begin{cases}7 n+1 & \text { if } i=0 \\ 2 n+2+2 i & \text { if } 1 \leq i \leq \frac{n-1}{2} \\ 8 n+4 & \text { if } i=\frac{n+1}{2} \\ 4 n+2 i & \text { if } \frac{n+3}{2} \leq i \leq n\end{cases}
$$

In view of the above labeling pattern and by the same way in Case (1), we have $f^{*}(x)=0, f^{*}(y)=7 n+1, f^{*}\left(v_{0}\right)=n+1, f^{*}\left(v_{\frac{n+1}{2}}\right)=n-1$ and $f^{*}\left(v_{n}\right)=5 n-7$.

Finally, $\quad f^{*}\left(v_{i}\right)= \begin{cases}(5 n+3+2 i) \bmod (8 n+4) & \text { if } \quad 1 \leq i \leq \frac{n-1}{2} ; \\ (n-7+2 i) \bmod (8 n+4) & \text { if } \quad \frac{n+3}{2} \leq i \leq n-1 .\end{cases}$

Hence, the labels of the vertices $v_{1}, v_{2}, v_{3}, \cdots, v_{\frac{n-3}{2}}, v_{\frac{n-1}{2}}$ will be $5 n+5,5 n+7,5 n+9 \cdots, 6 n, 6 n+2$, respectively. Also, the labels of the vertices $v_{\frac{n+3}{2}}, v_{\frac{n+5}{2}}, \cdots, v_{n-2}, v_{n-1}$ will be $2 n-4,2 n-2, \cdots, 3 n-11,3 n-9$, respectively. It is clear that $f^{*}(x)$, $f^{*}(y)$ and $f^{*}\left(v_{0}\right)$ are even and different from all the labels of the vertices $v_{i}$. 
Obviously, the vertex labels are all even and distinct. Also $f^{*}(x)$ and $f^{*}(y)$ are even and different from all the labels of the vertices $v_{i}$. Thus, the graph $\bar{K}_{2}+W_{n}$ is an edge even graceful graph for all $n$.

Illustration: In Fig. 7, we present an edge even graceful labeling of $\bar{K}_{2}+W_{10}$ and $\bar{K}_{2}+W_{11}$.

\section{Edge even graceful labeling of the double cone $\overline{\boldsymbol{K}}_{\mathbf{2}}+\boldsymbol{C}_{\boldsymbol{n}}$}

Theorem 8 The double cone $\bar{K}_{2}+C_{n}$ has an edge even graceful labeling for all $n$.

Proof Let $\{x, y\}$ be the vertices of $\bar{K}_{2}$ and $\left\{v_{1}, v_{2}, \cdots, v_{n}\right\}$ be the vertices of the graph $C_{n}$ so the edges are $\left\{x v_{i}, y v_{i}, v_{i} v_{i+1}, i=1,2, \cdots, n\right\}$. Let us use the standard notation $p=\left|V\left(\bar{K}_{2}+C_{n}\right)\right|=n+2$ and $q=\left|E\left(\bar{K}_{2}+C_{n}\right)\right|=3 n$. There are three cases:

Case (1): When $n \equiv 1(\bmod 6)$ or $n \equiv 3(\bmod 6)$. We define the labeling $f: E\left(\bar{K}_{2}+C_{n}\right) \longrightarrow\{2,4, \cdots, 6 n\}$ as follows:

$$
\begin{gathered}
f\left(x v_{i}\right)=2 i \quad \text { for } i=1,2, \cdots n, \\
f\left(y v_{i}\right)=6 n-2 i \quad \text { for } i=1,2, \cdots n,
\end{gathered}
$$

and

$$
f\left(v_{1} v_{n}\right)=6 n, \quad f\left(v_{i} v_{i+1}\right)=2 n+2 i \quad \text { for } i=1,2, \cdots n-1,
$$

The induced vertex labels are

$f^{*}(x)=\left[\sum_{i=1}^{n} f\left(x v_{i}\right)\right] \bmod (6 n)=\left[\sum_{i=1}^{n}(2 i)\right] \bmod (6 n)=\left(n^{2}+n\right) \bmod (6 n)$, If $n \equiv 1(\bmod 6) \Rightarrow 2 q=6 n=36 k+6$, then

$f^{*}(x) \equiv[12 k+2] \bmod (36 k+6)=2 n$.

If $n \equiv 3(\bmod 6) \Rightarrow 2 q=6 n=36 k+18$, then

$f^{*}(x)=\equiv[24 k+12] \bmod (36 k+12)=4 n$.

Similarly, $f^{*}(y)=\left[\sum_{i=1}^{n} f\left(y v_{i}\right)\right] \bmod (6 n)=\left[\sum_{i=1}^{n}(6 n-2 i)\right] \bmod (6 n)$. $\therefore f^{*}(y)=\left(5 n^{2}-n\right) \bmod (6 n)= \begin{cases}4 n & \text { if } n \equiv 1(\bmod 6) \\ 2 n & \text { if } n \equiv 3(\bmod 6) .\end{cases}$

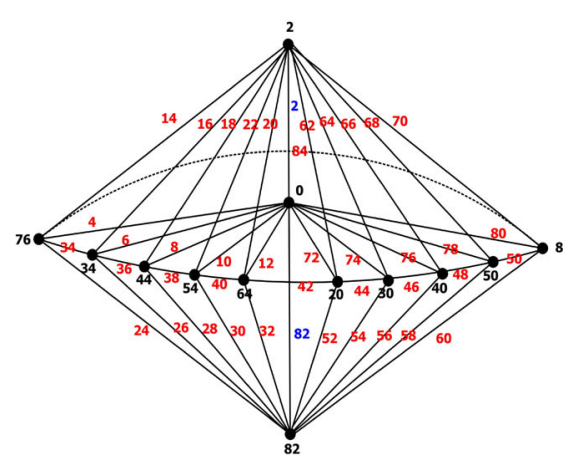

(a) $\bar{K}_{2}+W_{10}$

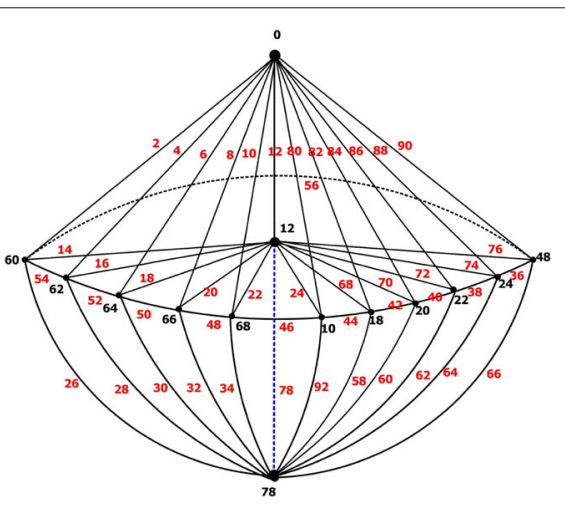

(b) $\bar{K}_{2}+W_{11}$

Fig. 7 An edge even graceful labeling of the graphs $\bar{K}_{2}+W_{10}$ and $\bar{K}_{2}+W_{11}$ 


$$
\begin{aligned}
f^{*}\left(v_{1}\right)=[ & \left.f\left(v_{1} v_{2}\right)+f\left(v_{n} v_{1}\right)+f\left(x v_{1}\right)+f\left(y v_{1}\right)\right] \bmod (6 n)=2 n+2, \\
f^{*}\left(v_{i}\right) & =\left[f\left(v_{i} v_{i+1}\right)+f\left(v_{i-1} v_{i}\right)+f\left(x v_{i}\right)+f\left(y v_{i}\right)\right] \bmod (6 n) \\
& =\left[f\left(v_{i} v_{i+1}\right)+f\left(v_{i-1} v_{i}\right)\right] \bmod (6 n) \\
& =(4 n+4 i-2) \bmod \quad(6 n), \quad 2 \leq i \leq n-1,
\end{aligned}
$$

and

$$
f^{*}\left(v_{n}\right)=\left[f\left(v_{n} v_{1}\right)+f\left(v_{n-1} v_{n}\right)+f\left(x v_{n}\right)+f\left(y v_{n}\right)\right] \bmod (6 n)=4 n-2 .
$$

Hence, the labels of the vertices $v_{1}, v_{2}, v_{3}, \cdots, v_{\frac{n-1}{2}}$ will be

$2 n+2,4 n+6,4 n+10, \cdots, 6 n-4$, respectively, and the labels of the vertices $v_{\frac{n+1}{2}}, v_{\frac{n+3}{2}}, \cdots, v_{n-1}, v_{n}$ will be $0,4, \cdots, 4 n-6,4 n-2$, respectively. Clearly, $f^{*}(x)$ and $f^{*}(y)$ are different from all the labels of the vertices $v_{i}$.

Case (2): When $n \equiv 5(\bmod 6)$, we define the labeling $f$ as follows:

$$
\begin{array}{crr}
f\left(y v_{i}\right)=2 n+2 i & \text { for } i=1,2, \cdots n, \\
f\left(v_{1} v_{n}\right)=4 n+2, & f\left(v_{i} v_{i+1}\right)=6 n-2 i+2
\end{array} \text { for } i=1,2, \cdots n-1,
$$

and

$f\left(x v_{i}\right)= \begin{cases}2 & \text { if } i=1 \\ 2 n-2 i+4 & \text { if } i=2,3, \cdots n\end{cases}$

Since $n \equiv 5(\bmod 6) \Rightarrow n=6 k+5 \Rightarrow 2 q=6 n=36 k+30$.

Then the induced vertex labels are

$f^{*}(y)=\left[\sum_{i=1}^{n}(2 n+2 i)\right] \bmod (6 n)=\left(3 n^{2}+n\right) \bmod (6 n)$

$$
\equiv\left[24\left(\frac{n-5}{6}\right)+20\right] \bmod (6 n)=4 n,
$$

$f^{*}(x)=\left(n^{2}+n\right) \bmod (6 n) \equiv 0, \quad f^{*}\left(v_{1}\right)=6 \quad$ and

$f^{*}\left(v_{i}\right)=(4 n-4 i+10) \bmod (6 n) \quad$ for $2 \leq i \leq n$.

Hence the labels of the vertices $v_{1}, v_{2}, v_{3}, \cdots, v_{\frac{n-1}{2}}, v_{\frac{n+1}{2}}, v_{\frac{n+3}{2}}, \cdots, v_{n-1}, v_{n}$ are $6,4 n+2,4 n-2, \cdots, 2 n+14,2 n+8,2 n+4, \cdots, 14,10$, respectively. Clearly, $f^{*}(x)$ and $f^{*}(y)$ are even and different from all the labels of the vertices $v_{i}$. Thus, the graph $\bar{K}_{2}+C_{n}$ is an edge even graceful graph.

Case (3): When $n$ is even, $n \geq 4$, we define the labeling function $f$ as follows:

$$
\begin{aligned}
& f\left(v_{1} v_{n}\right)=4 n, \quad f\left(v_{i} v_{i+1}\right)=2 n+2 i \quad \text { for } i=1,2, \cdots n-1, \\
& f\left(x v_{i}\right)= \begin{cases}2 i & \text { if } i=1,2, \cdots, \frac{n}{2} \\
4 n+2(i-1) & \text { if } \quad \frac{n}{2}+1 \leq i \leq n,\end{cases} \\
& \text { and }
\end{aligned}
$$

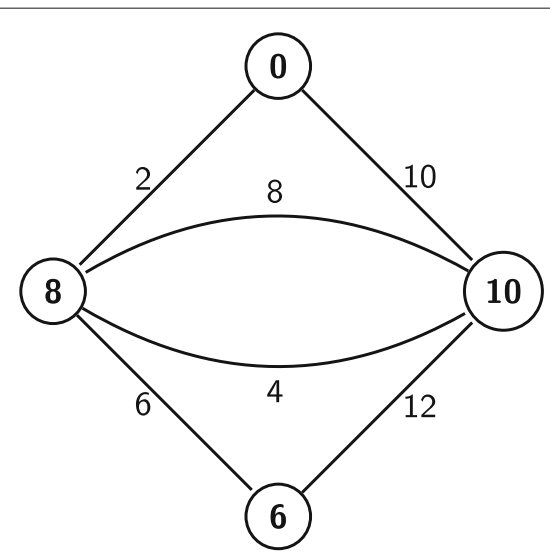

Fig. 8 An edge even graceful labeling of the double cone $\bar{K}_{2}+C_{2}$ 


$$
f\left(y v_{i}\right)= \begin{cases}2 n-2(i-1) & \text { if } 1 \leq i \leq \frac{n}{2} \\ 6 n & \text { if } i=\frac{n}{2}+1 \\ 6 n-2(i-1) & \text { if } \frac{n}{2}+2 \leq i \leq n\end{cases}
$$

Thus, the induced vertex labels are

$$
\begin{aligned}
f^{*}(x) & =\left[\sum_{i=1}^{n} f\left(x v_{i}\right)\right] \bmod (6 n)=0, \\
f^{*}(y) & =\left[\sum_{i=1}^{n} f\left(y v_{i}\right)\right] \bmod (6 n)= \\
{\left[f\left(y v_{1}\right)+f\left(y v_{n}\right)\right] \bmod (6 n)=2 n, } & \\
f^{*}\left(v_{1}\right) & =\left[f\left(x v_{1}\right)+f\left(y v_{1}\right)+f\left(v_{1} v_{2}\right)+f\left(v_{n} v_{1}\right)\right] \bmod (6 n)=2 n+4,
\end{aligned}
$$

and

$$
f^{*}\left(v_{i}\right)= \begin{cases}4 n-4 i+8 & \text { if } 2 \leq i \leq \frac{n}{2} \\ 5 n+2 & \text { if } i=\frac{n}{2}+1 \\ 6 n-4 i+6 & \text { if } \frac{n}{2}+2 \leq i \leq n .\end{cases}
$$

Hence, the labels of the vertices $v_{1}, v_{2}, v_{3}, \cdots, v_{\frac{n}{2}-1}, v_{\frac{n}{2}}$ are $2 n+4,4 n, 4 n-4, \cdots, 2 n+$ $12,2 n+8$, respectively, and the labels of the vertices $v_{\frac{n}{2}+1}, v_{\frac{n}{2}+2}, v_{\frac{n}{2}+3}, \cdots, v_{n-1}, v_{n}$ are $5 n+2,4 n-2,4 n-6, \cdots, 2 n+10,2 n+6$, respectively. Obviously the vertex labels are all even and distinct. Also, $f^{*}(x)$ and $f^{*}(y)$ are even and different from all the labels of the vertices $v_{i}$. Thus, the double cone $\bar{K}_{2}+C_{n}$ is an edge even graceful labeling when $n$ is even.

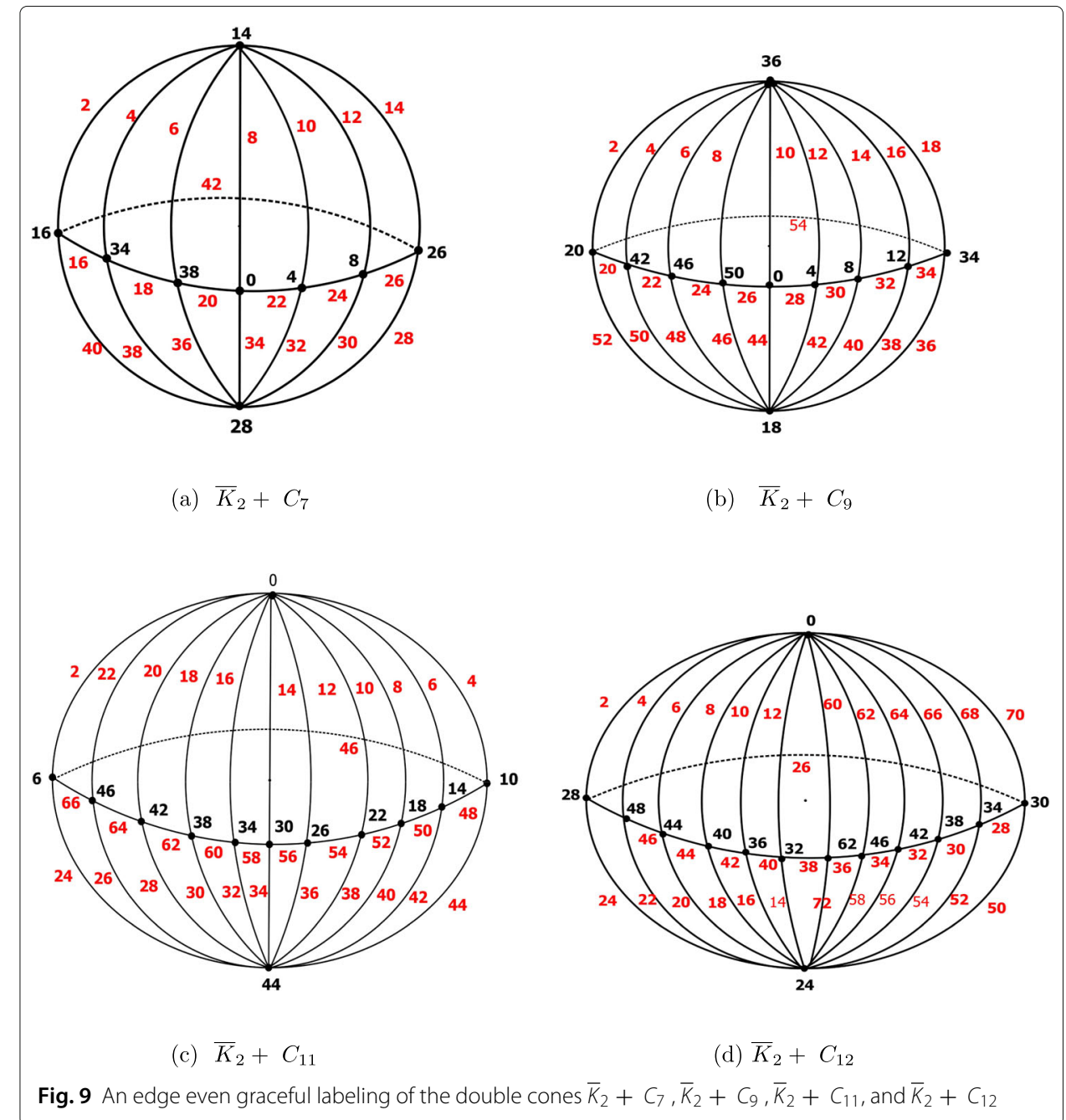


- If $n=2$, the double cone $\bar{K}_{2}+C_{2}$ has an edge even graceful labeling, see the following Fig. 8.

Illustration: In Fig. 9, we present an edge even graceful labeling of $\bar{K}_{2}+C_{7}, \bar{K}_{2}+C_{9}, \bar{K}_{2}+C_{11}$ and $\bar{K}_{2}+C_{12}$

\section{Acknowledgements}

The authors wish to express their thanks to the reviewers for the insightful comments and suggestions which have improved aspects of the work.

\section{Authors' contributions}

The authors read and approved the final manuscript.

Funding

The research for this manuscript is not funded.

\section{Availability of data and materials}

Not applicable.

\section{Competing interests}

The authors declare that they have no competing interests.

\section{Author details}

${ }^{1}$ Department of Mathematics, Faculty of Science, Suez University, Suez, Egypt. ${ }^{2}$ Department of Mathematics, Faculty of Science, Port-Said University, Egypt.

Received: 24 September 2019 Accepted: 2 March 2020

Published online: 12 May 2020

\section{References}

1. Gross, J., Yellen, J.: Graph theory and its applications. CRC Press, London (1999)

2. Bloom, G. S., Glomb, S. W.: Application of numbered undirected graphs. Proc. IEEE. 65, 562-570 (1977)

3. Acharya, B. D., Arumugam, S., Rosa, A.: Labeling of discreate strucutures and applications. Narosa Publishing House, New Delhi (2008)

4. Elsonbaty, A., Daoud, S. N.: Edge even graceful labeling of some path and cycle- related graphs. Ars Combinatoria. 130, 79-96 (2017)

5. Zeen El Deen, M. R.: Edge -even graceful labeling of some graphs, Vol. 27:20 (2019). https://doi.org/10.1186/s42787019-0025-x

6. Elsonbaty, A., Daoud, S. N.: Edge even graceful labeling of cylinder grid graphs. Symmetry. 11, 584 (2019). https:// doi:10.3390/sym11040584

7. Daoud, S. N.: Edge even graceful labeling of polar grid graphs. Symmetry. 11, 38 (2019). https://doi.org/10.3390/ sym 11010038

8. Zeen El Deen, M. R., Omar, N.: r - Edge-even graceful labeling of graphs. Ars Combinatoria. In press

9. Gallian, J. A.: A Dynamic survey of graph labeling. Electron. J. Comb. (2015). http://link.springer.com/10.1007/978-184628-970-5

\section{Publisher's Note}

Springer Nature remains neutral with regard to jurisdictional claims in published maps and institutional affiliations.

\section{Submit your manuscript to a SpringerOpen ${ }^{\odot}$ journal and benefit from:}

- Convenient online submission

- Rigorous peer review

- Open access: articles freely available online

- High visibility within the field

- Retaining the copyright to your article

Submit your next manuscript at $\gg$ springeropen.com 